\title{
PRICE-DISPERSED PREFERENCES AND C MEAN DEMAND
}

\author{
Egbert DIERKER, Hildegard DIERKER and Walter TROCKEL* \\ University of Bonn, 53 Bonn, Federal Republic of Germany \\ Received February 1982, accepted December 1983
}

In this paper we introduce the concept of price-dispersed preferences. Moreover we state conditions under which economies with price-dispersed preference distributions have a continuously differentiable mean demand function.

\section{Introduction}

The purpose of this article is to present a class of consumption sectors which exhibit a continuously differentiable mean demand although individual preferences are not assumed to be convex. As we have argued in an earlier paper [Dierker et al. (1980b, introduction)], several economic questions require mean demand to be a continuously differentiable function and not only to be a continuous one.

The literature on smoothing demand by aggregation shows that $\mathrm{C}^{0}$ mean demand functions can be obtained by methods which are not appropriate to obtain a $\mathrm{C}^{1}$ mean demand. Studying the differentiability of mean demand one encounters difficult conceptual problems which do not occur in the study of $\mathrm{C}^{0}$ mean demand. In the present paper we present a class of consumption sectors, i.e., of distributions of consumers' characteristics, with $\mathrm{C}^{1}$ mean demand. Presenting this class we use assumptions which are not generic. We think, though, that some of the methods used here are promising tools for the development of a more satisfactory theory of smoothing demand.

It is essential for the purpose of smoothing demand by aggregation in the presence of preference non-convexities that preferences are sufficiently dispersed. Since in a Euclidean space Lebesgue measure is often used to formalize dispersion, one is tempted to use Lebesgue measure in order to formulate the notion of dispersion on the space of preferences. This idea has led to the socalled parametric approach in which consumers are supposed to have characteristics in a finite dimensional space [see, in particular, Araujo and Mas-Colell (1978), Hildenbrand (1980), Sondermann (1975, 1976, 1980) and Yamazaki (1980)].

\footnotetext{
*We wish to thank H. Haller for many helpful discussions.
} 
In the present paper we formulate the notion of preference dispersion without stipulating that consumers' preferences form a finite dimensional space.

This paper is based on our previous work on the aggregation of demand derived from non-convex preferences [Dierker et al. $(1980 \mathrm{a}, \mathrm{b})]$, where we have exploited the fact that the space of consumers' characteristics is the product of the wealth space, which is a subset of the real line, and of the preference space, which has no Euclidean structure. The product structure of the measure space of consumers' characteristics lends itself to an application of Fubini's theorem: we integrate demand first with respect to wealth, then with respect to preferences.

In the first step of aggregation we require the wealth distribution, given any fixed preference, to have a continuous density with respect to Lebesgue measure. This condition is sufficient to obtain, in the first step, a continuous function which is continuously differentiable except on a closed null set of prices [cf. Dierker et al. (1980b)]. However, without further assumptions on the distribution of preferences, the second step, integration with respect to preferences, need not yield a differentiable mean demand.

In the present paper we deal with the second step, integration with respect to preferences, maintaining our Fubini type approach to the problem. We proceed as follows. We want to express the idea that similar demand behavior occurs in similar budget situations with similar probabilities. A budget situation being described by a price-wealth pair, we vary budget situations by varying prices, since we have already considered the variation of wealth in our previous work [Dierker et al. (1980b)].

A price system can be considered as a linear operator on the commodity space, stretching the axes of the commodity space, resp. 'rotating' the indifference hypersurfaces in the commodity space. Stretching the axes of the commodity space defines an action of the group $S$ of prices on the commodity space. ${ }^{1}$ The action of prices on the commodity space induces an action on preferences, which, roughly speaking, 'rotates' indifference hypersurfaces. The Haar measure on the group of price systems is employed to describe the notion of price-dispersed preferences with the aid of the action of price systems on preferences.

Price-dispersed preferences exhibit a qualitatively similar behavior of demand at different prices. This means that certain phenomena which destroy differentiability of demand, integrated with respect to wealth, namely vanishing Gaussian curvature of indifference hypersurfaces, critical jumps, and non-critical multiple jumps, are dispersed with respect to prices.

We say that a jump occurs if at least two different commodity bundles are demanded at the same budget situation. A jump is critical, if the marginal

\footnotetext{
${ }^{1}$ In a related context similar transformations have been used by Mas-Colell and Neuefeind (1977, p. 597).
} 
utilities of two points in the demand set coincide. A jump is called multiple, if there are more than two points in the demand set. Critical jumps lead to infinite slopes of the demand aggregated with respect to wealth keeping the preference fixed. Non-critical multiple jumps lead to kinks with finite slopes.

In this paper we do not study the problem of vanishing Gaussian curvature. A special case of this problem has been studied in Dierker et al. (1980b). Here we concentrate on multiple and critical jumps. Since, in our model, critical jumps occur as singularities of corank 1 in the price-wealth space, it is natural to assume that they are of a certain 'stable' type, namely, that they are Morin singularities [cf. Golubitsky and Guillemin (1973, p. 177)]. This assumption together with the price-wealth disperson of consumers' characteristics is essential for deriving the differentiability of mean demand.

The possible types of singularities of a mapping depend on the dimensions of the spaces related by the mapping: larger spaces allow more complicated singularities. We feel that this makes a major difference between the parametric approach and ours. The basic objects in the parametric approach are consumption sectors, the basic objects in our approach are individual consumers' characteristics. As a consequence, the singularities possibly encountered in the parametric approach depend on the number of commodities as well as on the number of parameters of the model, whereas they do only depend on the number of commodities in our approach. We would like to point out, thercforc, that it does make a difference for the problem of smoothing demand by aggregation whether one considers individual consumer characteristics as basic objects or not.

The paper is organized as follows: section 2 contains the model and the result, section 3 sketches the main idea of the paper in some examples, and section 4 contains the proofs.

\section{Model and result}

There are $l \geqq 2$ perfectly divisible commodities. The commodity space is $\mathbb{R}^{l}$. Every consumer's consumption set is

$$
X=\left\{x \in \mathbb{R}^{l} \mid x \gg>0\right\}
$$

The price space is

$$
S=\left\{p \in \mathbb{R}^{l} \mid p \gg 0, p_{l}=1\right\} .
$$

The use of commodity $l$ as a numéraire allows us to express the concept of price-dispersion in a convenient way. Occasionally we will identify $S$ with $\mathbb{R}_{+}^{l-1}$. 
We consider utility functions $u: X \rightarrow \mathbb{R}$ of class $\mathrm{C}^{\infty}$ satisfying

(U.1) $\mathrm{D} u(x) \gg 0$ for all $x \in X$,

(U.2) for every $x \in X$, the closure $\operatorname{cl}\left(u^{-1}(u(x))\right)$ of the indifference hypersurface through $x$ is contained in $X$,

(U.3) $g_{u}: X \rightarrow S$, defined by $g_{u}(x)=\mathrm{D} u(x)\left(\mathrm{D} u(x)_{l}\right)^{-1}$ has maximal rank, $l-1$, everywhere.

The set $\hat{\mathscr{P}}$ of all $\mathrm{C}^{\infty}$ utility functions satisfying ( $\left.\mathscr{U} .1\right),(\mathscr{U} .2)$ and $(\mathscr{U} .3)$, is endowed with the topology of uniform $\mathrm{C}^{\infty}$ convergence on compact sets. Every utility function $u \in \widehat{\mathscr{P}}$ defines a unique preference relation, $\lesssim$, on $x$ by

$$
x \lesssim y \Leftrightarrow u(x) \leqq u(y) .
$$

Each utility function $u$ obcying this condition represents the samc preference relation $\lesssim$. Obviously, $g_{u}$, as defined in ( $\left.\mathscr{U} .3\right)$, does only depend on the preference relation $\lesssim$ rather than on $u$. Therefore, we will replace $g_{u}$ by $g_{\precsim}$, or simply by $g$, if no confusion is possible.

Let $\mathscr{P}$ be the set of preference relations representable by utility functions in $\widehat{P}$. We endow the set $\mathscr{P}$ with the identification topology of the projection $\widehat{\mathscr{P}} \rightarrow \mathscr{P}$. This topology on $\mathscr{P}$ is the topology of $\mathrm{C}^{\infty}$ uniform convergence on compact sets of the maps $g_{\lessgtr}$. [cf. Mas-Colell (1978, sect. 4)]. It makes $\mathscr{P}$ a Polish space, i.e., a separable space whose topology can be generated by a complete metric. For an excellent comprehensive treatment of topological spaces of smooth preferences we refer to Mas-Colell (1976).

We will consider measure spaces of consumers. Each consumer is characterized by his preference, $\lesssim \in \mathscr{P}$, and by his wealth, $w \in] 0, \infty[$. The demand set at the price system $p \in S$ of a consumer with characteristics $(\precsim, w) \in \mathscr{P} \times] 0, \infty[$ is

$$
\phi(\lesssim, p, w)=\{x \in X \mid p x \leqq w, x \lesssim y \Rightarrow p y>w\} .
$$

The demand correspondence

$$
\phi: \mathscr{P} \times S \times] 0, \infty[\rightarrow X,
$$

defined in this way, is upper-hemicontinuous, abbreviated u.h.c. [Mas-Colell (1977, p. 1392)].

The distribution of consumers' characteristics is a probability, $\tau$, on the space $\mathscr{P} \times] 0, \infty[$.

Let $\mathscr{B}(T)$ denote the $\sigma$-field of Borel sets on a topological space $T$.

Since for every $p \in S$ we have

$$
\text { graph } \phi(\cdot, p, \cdot) \in \mathscr{B}(\mathscr{P} \times] 0, \infty[) \otimes \mathscr{B}(X),
$$


we define mean demand at prices $p$ for the given distribution $\tau$ of consumption characteristics by

$$
F(p)=\int_{\mathscr{P} \times] 0, \infty[} \phi(\lesssim, p, w) \mathrm{d} \tau
$$

For a detailed treatment of integration of correspondences we refer to Hildenbrand (1974).

The smoothing effect of aggregation relies on a suitable dispersion of consumers' characteristics. A suitable dispersion of consumers' characteristics should be reflected in a suitably dispersed demand behavior. Hence we are looking for a probability on $\mathscr{P} \times] 0, \infty[$ with, roughly speaking, the following property: in similar budget situations, similar demand behavior occurs with similar probabilities.

To make this requirement precise we have to specify what we mean by similar demand behavior at similar budget situations. For that purpose we model the space of budgets, i.e., price-wealth pairs, as a group operating on consumers' characteristics. Due to the use of a numéraire the action of prices does not affect wealth and the action of wealth does not affect preferences. Therefore, following the presentation in our earlier work [Dierker et al. (1980b)], we will formalize wealth dispersion and price dispersion separately.

Accordingly, we represent the distribution $\tau$ on $\mathscr{P} \times] 0, \infty[$ as composed of distributions on $\mathscr{P}$ and on $] 0, \infty[$. More precisely, we write

$$
\tau=\int_{\mathscr{P}} \delta_{\lessgtr} \mu(\mathrm{d} \lesssim)
$$

[cf. Dierker et al. (1980b)]. The probability $\mu$ is the image measure of $\tau$ under the projection of $\mathscr{P} \times] 0, \infty\left[\right.$ onto $\mathscr{P}$; the probabilities $\delta_{\preccurlyeq}$ live on the fibres $\{\precsim\} \times] 0, \infty[, \precsim \in \mathscr{P}$. We make assumptions on $\tau$ by making assumptions on $\mu$ and on $\delta_{\lessgtr}$.

First we will formalize price dispersion of preferences as a property of $\mu$. This requires us to define the action of $S$ on $\mathscr{P}$.

Consider the price space $S$ with the following multiplication:

$$
S \times S \rightarrow S:\left(q, q^{\prime}\right) \mapsto q \circ q^{\prime}=\left(q_{1} \cdot q_{1}^{\prime}, \ldots, q_{l-1} \cdot q_{l-1}^{\prime}, 1 \cdot 1\right) .
$$

With this multiplication the price space $S$ becomes a locally compact, $\sigma$ compact, commutative group. Hence there exists a measure on $(S, \mathscr{B}(S))$, invariant under the group operation, which is unique up to normalization. Such a measure is called a Haur measure.

Next we consider the following actions of the group $S$ of prices on the consumption set $X$ and on the space $\mathscr{P}$ of preferences:

$$
X \times S \rightarrow X:(x, q) \mapsto x \circ q=\left(x_{1} \cdot q_{1}, \ldots, x_{l-1} \cdot q_{l-1}, x_{l} \cdot 1\right),
$$




$$
a: \mathscr{P} \times S \rightarrow \mathscr{P}:(\precsim, q) \mapsto \precsim_{q}=\{(x \circ q, y \circ q) \in X \times X \mid x \precsim y\}
$$

By the commutativity of the acting group $S$ we have for every $q, q^{\prime} \in S$ and for every $\lesssim \in \mathscr{P}$

$$
\left(\lesssim_{q}\right)_{q^{\prime}}=\lesssim_{q \circ q^{\prime}}=\left(\lesssim_{q^{\prime}}\right)_{q}
$$

Note that the numeraire commodity $l$ is not affected by the action $(x, q) \mapsto x \circ q$. Hence, wealth change and price change are kept apart.

The action $a$ is continuous, hence measurable. Moreover it maps $\mathscr{P} \times S$ onto $\mathscr{P}$. Hence, by the disintegration theorem [Parthasarathy (1967, Theorem 8.1 , p. 147)], every probability $\gamma$ on $\mathscr{P} \times S$ has a unique disintegration

$$
\gamma=\int_{\mathscr{P}} \xi_{\lessgtr \gamma}^{\prime} \gamma \circ a^{-1}(\mathrm{~d} \precsim)=\int_{\mathscr{P}} \xi_{\lessgtr}^{\prime} \mu^{\prime}(\mathrm{d} \precsim)
$$

The probability $\mu^{\prime}$ is the image measure of $\gamma$ under the map $a$, the probabilities $\xi_{\lesssim}^{\prime}$ on $\mathscr{P} \times S$ live on the fibres $a^{-1}(\precsim)$ over $\lesssim, \precsim \in \mathscr{P}$. For every $\lesssim \in \mathscr{P}$ the measurable bijective map $q \rightarrow\left(\lesssim_{q-1}, q\right)$ from $S$ onto

$$
a^{-1}(\lesssim)=\left\{\left(\lesssim^{\prime}, q\right) \in \mathscr{P} \times S \mid \lesssim_{q}^{\prime}=\precsim\right\}=\left\{\left(\lesssim_{q-1}, q\right) \mid q \in S\right\}
$$

allows us to consider the Haar measure on $S$ as living on $a^{-1}$ (Љ) without mentioning explicitly the transformation formula for measures.

Clearly, the measures $\gamma$ and $\xi_{\Im}^{\prime}$, $\lesssim \in \mathscr{P}$, have image measures under the projection from $\mathscr{P} \times S$ restricted to $a^{-1}(\lesssim)$ onto $\mathscr{P}$. This gives us the following commutative diagram:

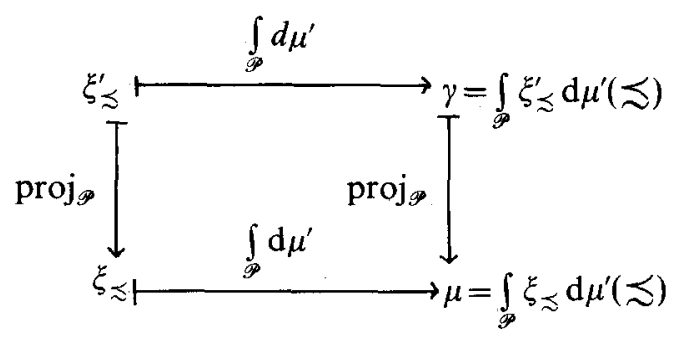

defining probabilities $\xi_{\precsim}$ on the orbits $\{\precsim\} \circ S=\left\{\S_{q} \mid q \in S\right\}, \precsim \in \mathscr{P}$, as image measures of $\xi_{\precsim}^{\prime}$ under proj $\left.\right|_{a-1(\lesssim)}$.

Definition. A probability $\mu$ on $(\mathscr{P}, \mathscr{B}(\mathscr{P}))$ is called price-dispersed iff $\mu$ is the marginal distribution on $(\mathscr{P}, \mathscr{B}(\mathscr{P}))$ of a probability $\gamma$ on $(\mathscr{P} \times S, \mathscr{B}(\mathscr{P} \times S))$ 
with disintegration

$$
\gamma=\int_{\mathscr{P}} \xi_{\precsim}^{\prime} \mathrm{d} \mu^{\prime}(\precsim)
$$

where $\xi_{\lessgtr}^{\prime}$ is absolutely continuous with respect to the Haar measure on $S$ for $\mu^{\prime}$-almost every preference $\lesssim$.

In an analogous way one could define wealth-dispersion by using the action $] 0, \infty[\times] 0, \infty[\rightarrow] 0, \infty[,(v, w) \mapsto v \cdot w$. However, in this simple framework it appears to be appropriate to directly state the following equivalent definition.

Definition. A probability $\delta_{\precsim}$ on $(\{\precsim\} \times] 0, \infty[, \mathscr{B}(\{\precsim\} \times] 0, \infty[0)$ is called wealth-dispersed iff it is absolutely continuous with respect to Lebesgue measure $\lambda^{1}$.

In fact, we will make dispersion assumptions which are somewhat more restrictive than the definitions above, in particular, we will use compactness in various ways.

Let $\tau=\int_{\mathscr{P}} \delta_{\precsim} \mu(\mathrm{d} \precsim)=\int_{\mathscr{P}} \int_{\mathscr{P}} \delta_{\precsim} \xi_{\precsim}(\mathrm{d} \lesssim) \mu^{\prime}(\mathrm{d} \precsim)$. We assume:

$\left(\mathscr{M}_{\text {comp }}\right)$ supp $\mu^{\prime}$ is compact.

Furthermore, we want all the $\xi_{\lessgtr}^{\prime}$, $€ \operatorname{supp} \mu^{\prime}$, to be a bounded family of probabilities all having the same $L^{1}$-space as the Haar measure on some compact neighborhood of ideS. To make this point precise, let $\bar{B}$ be a compact neighborhood of id $\in S$. Let $\chi \in \mathscr{M}^{1}(\bar{B})$ be the restriction of a Haar measure to $\bar{B}$. For convenience, we will not distinguish between $\bar{B}$ and $\{\lesssim\} \circ \bar{B}$ and between $\mathscr{M}^{1}(\bar{B})$ and $\mathscr{M}^{1}(\{\lesssim) \circ \bar{B})$.

Definition. A probability $\mu$ on $(\mathscr{P}, \mathscr{B}(\mathscr{P}))$ is called uniformly, locally pricedispersed if for all neighborhoods $U$ of id $\in S$ there are a compact neighborhood $\bar{B}$ of id, $\bar{B} \subset U$, and a probability $\gamma$ on $(\mathscr{P} \times \bar{B}, \mathscr{B}(\mathscr{P} \times \bar{B}))$ such that

(i) $\quad \mu$ is the marginal distribution on $(\mathscr{P}, \mathscr{B}(\mathscr{P}))$ of $\gamma$ with disintegration $\gamma=\int_{\mathscr{P}} \xi_{\lesssim}^{\prime} \mu^{\prime}(\mathrm{d} \precsim)$, where $\mu^{\prime}=\gamma \circ\left(\left.a\right|_{\mathscr{P} \times \bar{B}}\right)^{-1}$;

(ii) for $\mu^{\prime}$-almost every $\precsim$, the probabilities $\xi_{\precsim}^{\prime}$ on $(\bar{B}, \mathscr{B}(\bar{B}))$ are absolutely continuous with respect to $\chi$;

(iii) the densities $\mathrm{d} \xi_{\lessgtr}^{\prime} / \mathrm{d} \chi$, $\lesssim \in \operatorname{supp} \mu^{\prime}$, are elements of some weak*-compact subset of $L^{\infty}(\bar{B}, \widetilde{B}(\bar{B}), \chi)$.

We assume:

$\left(\mathscr{M}_{p}\right)$ The probability $\mu$ on $(\mathscr{P}, \mathscr{B}(\mathscr{P}))$ is uniformly, locally price-dispersed. 
Remark. Roughly speaking, one might look at our concept of pricedispersion as being based on a decomposition of the space of preferences into two factors, the space $S$ of prices and the space $\mathscr{P} / S$ of equivalence classes $\precsim \circ S$. Price-dispersed probabilities in this case would be those which have marginal distributions on $S$ absolutely continuous with respect to a Haar measure on $S$ and any marginal distribution on $\mathscr{P} / S$. However, this formalization is unsatisfactory since there is no reason why orbits $\precsim \circ S$ should be closed and the $\sigma$-field generated by these orbits should be standard Borel. We should like to mention, though, that monotone preferences on the closed consumption set $\mathbb{R}_{+}^{I}$ give rise to closed orbits.

Our general concept is based on the disintegration theorem which extends the generalized Fubini theorem to situations where there is no a priori given product of measure spaces.

Next, we state the wealth-dispersion assumption which by its local and uniform character is very similar to assumption $\left(\mathscr{M}_{p}\right)$,

Let $\tau=\int_{\not \Rightarrow} \delta_{\precsim} \mu(\mathrm{d} \precsim)$. We assume:

$\left(\mathscr{M}_{w}\right)$ The probabilities $\delta_{\lesssim}$ are wealth-dispersed with continuous Lebesguedensities $h_{\precsim}, \precsim \in \operatorname{supp} \mu$. Moreover $\left\{h_{\precsim}\right\}_{\precsim \in \operatorname{supp} \mu}$ is a uniformly bounded family of continuous functions on $] 0, \infty[$ vanishing outside some compact interval $[w, \bar{w}] \subset] 0, \infty[$.

Remark. Without continuity of the densities $h_{\precsim}, \precsim \in \operatorname{supp} \mu$, there is no reason why demand aggregated with respect to wealth should be a continuously differentiable function of prices.

Assume $\left(\mathscr{M}_{\text {comp }}\right),\left(\mathscr{M}_{p}\right)$ and $\left(\mathscr{M}_{w}\right)$. Since supp $\mu^{\prime}$ and $\bar{B}$ are compact and $a$ is continuous, $a\left(\left(\operatorname{supp} \mu^{\prime}\right) \times \bar{B}\right)$ is compact. Hence supp $\gamma$ and, accordingly, supp $\mu$ are compact. Furthermore, $\operatorname{supp} \delta_{\precsim} \subset[w, \bar{w}]$ for all $\lesssim \in \operatorname{supp} \mu$. Finally, supp $\tau$ is a closed subset of $(\operatorname{supp} \mu) \times[\underline{w}, \bar{w}]$. This yields:

Remark. Under the assumptions $\left(\mathscr{M}_{\text {comp }}\right),\left(\mathscr{M}_{p}\right)$ and $\left(\mathscr{M}_{w}\right)$ the distribution $\tau$ of consumers' characteristics has a compact support $\operatorname{supp} \tau \subset \mathscr{P} \times] 0, \infty \mathrm{L}$.

Let us sketch the effect of the disintegration of $\tau$ and of $\mu$ on the demand formula. Mean demand at the price system $p$ is defined as

$$
F(p)=\int_{\mathscr{P} \times \mathrm{J} 0 . \infty[} \phi(\lesssim, p, w) \mathrm{d} \tau .
$$

Denote by $\Phi(\precsim, \cdot)$ the demand integrated with respect to wealth, i.e., for every $p \in S$,

$$
\Phi(\precsim, p)=\int_{0}^{\infty} \phi(\precsim, p, w) \mathrm{d} \delta_{\lessgtr}(w) .
$$


Using the price-dispersion of $\mu$ one obtains

$$
\begin{aligned}
& F(p)=\int_{\mathscr{P}} \Phi(\precsim, p) \mathrm{d} \mu(\precsim)=\int_{\mathscr{P}} \int_{\mathscr{P}} \Phi\left(\precsim^{\prime}, p\right) \mathrm{d} \xi_{\precsim}\left(\precsim^{\prime}\right) \mathrm{d} \mu^{\prime}(\precsim)
\end{aligned}
$$

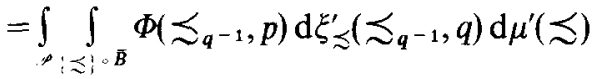

$$
\begin{aligned}
& =\int_{\mathscr{P} \bar{B}} \int_{\bar{B}} \Phi\left(\precsim_{q^{-1}}, p\right) \frac{\mathrm{d} \xi^{\prime}}{\mathrm{d} \chi}(q) \mathrm{d} \chi(q) \mathrm{d} \mu^{\prime}(\precsim) .
\end{aligned}
$$

In addition to the dispersion and compactness assumptions we make three assumptions on the support of $\tau$.

First, we exclude the case that the Gaussian curvature of an indifference hypersurface vanishes at a point in the demand set of some agent $(\precsim, w) \in \operatorname{supp} \tau$ at some price system $p$. This assumption will be made, because aggregation with respect to wealth which is not the subject of this paper, seems to be a promising way to treat vanishing curvature [cf. Dierker et al. (1980b, sect. 4)].

$\left(\mathscr{M}_{\text {curv }}\right) \quad$ For all $(\precsim, w) \in \operatorname{supp} \tau$, for all $p \in S$, and for all $x \in \phi(\lesssim, p, w)$ the Gaussian curvature of the $\lesssim$-indifference hypersurface through $x$ does not vanish at $x$.

The problem of vanishing Gaussian curvature assumed away, we concentrate on the problem of non-uniqueness of individual demand. Consider a fixed preference $\lesssim \in \mathscr{P}$, represented by a utility function $u$. Let

$$
X_{1}=\left\{x \in X \mid \text { Hessian of }\left.u\right|_{\mathrm{Ker} \mathrm{D} u(x)} \text { is negative definite }\right\} \text {. }
$$

For two different points $x, y \in X_{1}$ to be demanded at price system $p$ it is necessary that $u(x)=u(y), g(x)=g(y)=p, p x=p y$. These are $1+(l-1)+(l-1)$ $+1=2 l$ equations in the variables $p \in S$ and $(x, y) \in\left(X_{1} \times X_{1} \backslash\right.$ diagonal). The solutions of this system of equations form an $(l-1)$-dimensional $C^{\infty}$ submanifold, denoted $\Gamma_{\preccurlyeq}$, of $S \times\left(X_{1} \times X_{1} \backslash\right.$ diagonal) [cf. Lemma 2 in Dierker et al. (1980b, sect. 3)].

Lemma 1. Let $\lesssim \in \mathscr{P}$. Then the mapping $\left.i: \Gamma_{\preccurlyeq} \rightarrow S \times\right] 0, \infty[:(p, x, y) \mapsto(p, p x)$ is an immersion.

Individual demand $\phi(\lesssim, p, w)$ is a singleton depending smoothly on $(p, w)$ unless $(p, w) \in i\left(\Gamma_{\Im}\right)$. There are two phenomena which may cause trouble if one integrates demand, given $\precsim$, with respect to the wealth distribution $\delta_{\precsim}$ near the price system $p$. First, $p$ can be a critical value of $\pi \circ i: \Gamma_{\lessgtr} \rightarrow S$, where 
$\pi$ projects $i\left(\Gamma_{\S}\right)$ to $S$. Second, $i\left(\Gamma_{\S}\right)$ can cross itself above $p$. We say that a critical jump occurs in the first case and a multiple jump occurs in the second case. Both of these situations are illustrated in fig. 1.

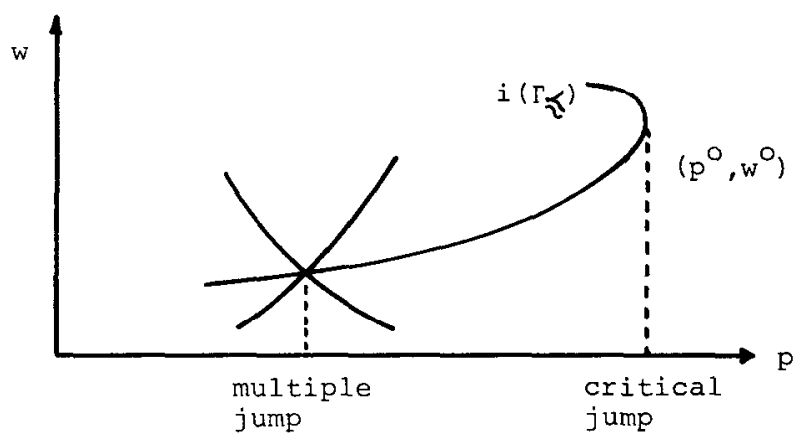

Fig. 1

Next we state some kind of normal crossings assumption on $i\left(\Gamma_{\precsim}\right)$. This assumption has been used in Dierker et al. $(1980 \mathrm{~b} \text {, Lemma } 4)^{2}$ to show that triple jumps form a manifold of dimension $l-2$. We assume:

$\left(\mathscr{M}_{n c}\right) \quad$ Let $(\lesssim, w) \in \operatorname{supp} \tau$ and let $u$ be a utility function representing $\lesssim$. Let $(x, y, z) \in X_{1} \times X_{1} \times X_{1}, \quad x \neq y, \quad x \neq z, \quad y \neq z, u(x)=u(y)=u(z)$. Assume $\alpha \mathrm{D} u(x)=\beta \mathrm{D} u(y)=\gamma \mathrm{D} u(z)=p, p x=p y=p z$, and $x-y=\lambda(y-z)$. Then $\lambda \neq(\alpha-\beta) /(\beta-\gamma)$.

Although critical and multiple jumps may occur simultaneously, we shall exclude such situations on $\operatorname{supp} \tau$. Let $\left(\lesssim^{0}, w^{0}\right) \in \operatorname{supp} \tau$. Let $\left(p^{0}, x^{0}, y^{0}\right) \in \Gamma_{\lesssim^{0}}$ be a critical point of $\pi \circ i: \Gamma_{\preccurlyeq 0} \rightarrow S$. We assume that no multiple jump occurs at $\left(p^{0}, w^{0}\right)$, where $w^{0}=p^{0} x^{0}=p^{0} y^{0}$. Then there is a neighborhood $U$ of $\left(p^{0}, w^{0}\right)$ in $S \times] 0, \infty\left[\right.$ such that $i\left(\Gamma_{\preccurlyeq 0}\right) \cap U$ is a manifold. It follows from rank assumption $(\mathscr{U} .3)$ that the corank of $\pi$ at $\left(p^{0}, w^{0}\right)$ is 1 . Therefore, one expects to find a fold if $l=2$, a fold or cusp if $l=3$, etc. That is to say, $i\left(\Gamma_{\Im^{0}}\right)$ sits in $S \times] 0, \infty\left[\operatorname{near}\left(p^{0}, w^{0}\right)\right.$ as the hypersurface

$$
\left.\Sigma^{k}=\left\{x_{1}, \ldots, x_{l}\right) \mid x_{1}+x_{2} x_{l}+x_{3} x_{l}^{2}+\cdots+x_{k} x_{l}^{k-1}+x_{l}^{k+1}=0\right\}, \quad k \leqq l-1,
$$

sits in $\mathbb{R}^{l-1} \times \mathbb{R}$. Moreover, changing the preference $\precsim^{0}$ slightly will not change this picture qualitatively. The reader is referred to the discussion of Morin singularities in Golubitsky and Guillemin (1973, ch. VII). We assume:

\footnotetext{
${ }^{2}$ There this assumption has been denoted (U5).
} 
$\left(\mathscr{M}_{\text {Morin }}\right) \quad$ Let $\left(\nwarrow^{0}, w^{0}\right) \in \operatorname{supp} \tau$. Let $\left(p^{0}, x^{0}, y^{0}\right) \in \Gamma_{\nwarrow^{0}}$ be a critical point of

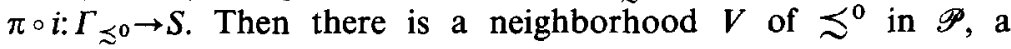
natural number $k \leqq l-1$, and a continuous mapping

$\mathscr{H}: V \rightarrow \mathrm{C}^{\infty}\left(\mathbb{R}^{1-1} \times \mathbb{R}, S \times\right] 0, \infty[): \precsim \mapsto H_{\preccurlyeq}, \quad$ such that

(i) $H_{\preccurlyeq 0}(0,0)=\left(p^{0}, w^{0}\right)$,

(ii) for any $\lesssim \in V, H_{\lessgtr}$ is a fibrewise diffeomorphism into $S \times] 0, \infty$ [, where fibrewise means that $H_{\Im}$ is of the form $H_{\S}\left(\hat{x}, x_{l}\right)=\left(H_{\lessgtr 1}^{1}(\hat{x}), H_{\Im 2}^{2}\left(\hat{x}, x_{l}\right)\right)$,

(iii) for any $\lesssim \in V$,

$$
\begin{aligned}
& H_{\S}\left(\left\{x \in \mathbb{R}^{l-1} \times \mathbb{R} \mid x_{1}+x_{2} x_{l}+x_{3} x_{l}^{2}+\cdots+x_{k} x_{l}^{k-1}+x_{l}^{k+1}=0\right\}\right) \\
& =H_{\S}\left(\Sigma^{k}\right)=i\left(\Gamma_{\S}\right) \cap \text { image } H_{\lessgtr} .
\end{aligned}
$$

It suffices for our purposes to endow $C^{\infty}\left(\mathbb{R}^{l-1} \times \mathbb{R}, S \times\right] 0, \infty[)$ with the topology of uniform $\mathrm{C}^{\infty}$-convergence on compact sets.

Formulating the assumption $\left(\mathscr{M}_{\text {Morin }}\right)$ we implicitly use the idea that 'the natural geometric forms are described by stable maps' [Bröcker and Lander $(1975$, p. 66)]. The special structure of these Morin singularities of ThomBoardman type $S \overbrace{1 \ldots 1}^{k} k \leqq l-1$, ('generalized cusps') of mapping between $(l-1)$-dimensional spaces allows us to visualize these singularities in $\mathbb{R}^{l-1} \times \mathbb{R}$. Assumption $\left(\mathscr{M}_{\text {Morin }}\right)$ takes advantage of the special structure of generalized cusps. This assumption, together with the notion of price-dispersed preferences, plays a crucial role in the proof of the central result of this paper.

Theorem. Let $\tau$ be a probability measure on $\mathscr{P} \times] 0, \infty[$ satisfying assumptions $\left(\mathscr{M}_{\text {comp }}\right),\left(\mathscr{M}_{p}\right),\left(\mathscr{M}_{w}\right),\left(\mathscr{M}_{\text {curv }}\right),\left(\mathscr{M}_{\mathrm{nc}}\right)$, and $\left(\mathscr{M}_{\text {Morin }}\right)$. Then the mean demand

is a $\mathrm{C}^{1}$ function.

$$
F: S \rightarrow X: p \mapsto \int_{g \times 10, \infty[} \phi(\precsim, p, w) \mathrm{d} \tau
$$

Here we would like to remark that the derivative of the mean demand $F$ is obtained by integrating the derivative of $\Phi(\lesssim, \cdot): S \rightarrow \mathbb{R}^{l}$ : $p \mapsto \int_{0}^{\infty} \phi(\lesssim, p, w) \delta_{\lesssim}(\mathrm{d} w)$ with respect to the distribution $\mu$ of preferences. Sonderman $(1976$, p. 422) gives an example explaining why interchanging the order of differentiation and integration fails, when one considers individual demand $\phi(\lesssim, p, w)$ instcad of $\Phi(\precsim, p)=\int_{0}^{\infty} \phi(\precsim, p, w) \delta_{\precsim}(\mathrm{d} w)$. Thus aggregation with respect to wealth, given $\lesssim$, is important when one wants to capture the influence of preference non-convexities on the derivative $\mathrm{D} F$ of mean demand.

The following lemma states a condition sufficient for interchanging the 
order of differentiation and integration. The lemma is a simple consequence of Lebesgue's dominated convergence theorem. Hence it is stated without proof. For closely related statements, see Bauer (1968, p. 211) or Schwartz (1967, p. 721).

Lemma 2. Let $\mu$ be a probability on $(\mathscr{P}, \mathscr{B}(\mathscr{P}))$. For $\mu$-almost every $\lesssim \in \mathscr{P}$ let $\Phi(\lesssim, \cdot): S \rightarrow \mathbb{R}^{l}$ be a function which is $\mathrm{C}^{1}$ at $p^{0}$. Suppose there exists a $\mu$ integrable function $j: \mathscr{P} \rightarrow \mathbb{R} \cup\{\infty\}$ and a neighborhood of $p^{0}$ such that for all $p$ in this neighborhood and for $\mu$-almost every $\lesssim \in \mathscr{P}$ we have

$$
\left\|\Phi\left(\precsim, p^{0}\right)-\Phi(\precsim, p)\right\| \leqq j(\precsim)\left\|p-p^{0}\right\| .
$$

Then $\int \Phi(\lesssim, \cdot) \mathrm{d} \mu$ is $\mathrm{C}^{1}$ at $p^{0}$ and

$$
\mathrm{D} \int \Phi\left(\precsim, p^{0}\right) \mathrm{d} \mu=\int \mathrm{D} \Phi\left(\precsim, p^{0}\right) \mathrm{d} \mu .
$$

To be specific, let $\|\cdot\|$ denote the Euclidean norm throughout this paper.

\section{Examples}

In this section we study the regularizing effect of price-dispersion of preferences in particularly simple cases. We shall consider a multiple, noncritical jump in an economy with two commodities and critical jumps in economies with two resp. three commodities (fold respectively cusp). To illustrate the effect of price-dispersion, we consider one preference $\lesssim \in \mathscr{P}$ and disperse it by the action of a compact neighborhood $\bar{B}$ of the identity in $S$, i.e., we study the case where $\mu$ is concentrated on $\precsim \circ \vec{B}$.

\subsection{Multiple, non-critical jump}

Imagine the preference pattern on $X \subset \mathbb{R}^{2}$ as depicted in fig. 2. To each indifference curve there belong three pairs of commodity bundles each of which forms a simple jump. To indifference curve $a^{\prime}$ there is associated a budget situation in which one of these pairs is demanded whereas the other two pairs are not demanded in any budget situation. Indifference curve $c^{\prime}$ exhibits two jumps which are demanded at corresponding price systems, whereas one pair is not demanded. The pairs on $c^{\prime}$ which are demanded at suitable budget situations are formed by points in the middle and on the left and by points in the middle and on the right. The only pair demanded on indifference curve $a^{\prime}$ is formed by points on the left and on the right. The indifference curve $b^{\prime}$ is exceptional. All three pairs are associated with the same budget situation. We say that the three points on the left, in the middle and on the right form a multiple jump. Fig. 3 illustrates the budget situations 


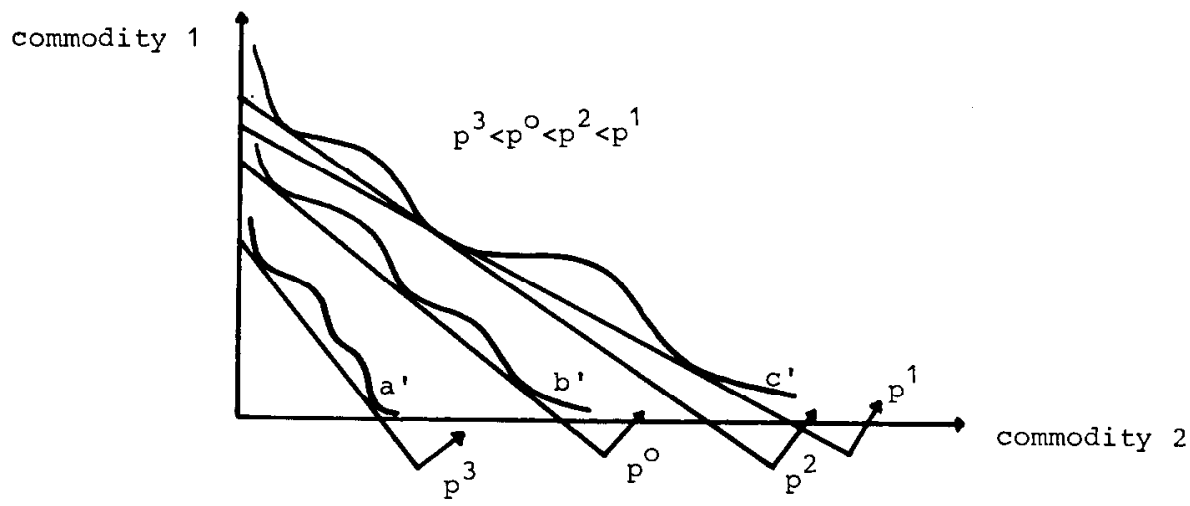

Fig. 2

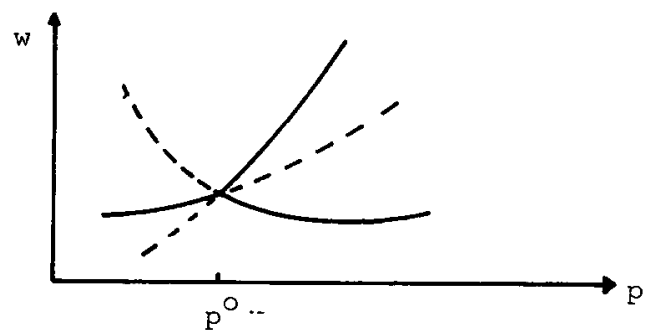

Fig. 3

at which a jump occurs. The solid lines correspond to jumps formed by demanded pairs, the dotted lines correspond to jumps formed by nondemanded pairs. For $p<p^{0}$ the demand aggregated with respect to wealth given $\lesssim$, i.e., $\Phi(\precsim):, S \rightarrow \mathbb{R}^{l}$, is the sum of two integrals of $C^{1}$ functions, where the upper boundary of the first integral is given by the solid line and coincides with the lower boundary of the second integral. The boundaries of integration depending differentiably on $p$, both integrals depend differentiably on $p$. Similarly, for $p>p^{0}$, the function $\Phi(\precsim, \cdot): S \rightarrow \mathbb{R}^{l}$ is the sum of three integrals, their boundaries being given by the two solid lines. The three integrals depend differentiably on $p$. At $p^{0}$ the function $\Phi(\precsim, \cdot): S \rightarrow \mathbb{R}^{l}$ exhibits a kink. Now let us vary the preference by stretching the vertical axis of the positive orthant. The linear mapping $\left.\left(x_{1}, x_{2}\right) \mapsto\left(x_{1} q, x_{2}\right), q \in\right] 0, \infty[$, leaves the wealth associated with any budget line unaltered, whereas the price associated with a budget line is multiplied by $q^{-1}$. Furthermore, a jump exhibited by $\lesssim$ before the action of $q$ takes place is transformed into a jump exhibited by $\precsim_{q}$ after the action has taken place. The function $\Phi\left(\lesssim_{q}, \cdot\right)$ has a kink at $p^{0} \circ q^{-1}$. Thus $\mu$-almost every preference is $C^{1}$ at $p^{0}$. The slopes of $\Phi\left(\lesssim_{q}, \cdot\right)$ stay uniformly bounded in a neighborhood of $p^{0}$. Consequently, 
there is an integrable Lipschitz constant for $\Phi\left(\lesssim_{q}, \cdot\right), q \in S$, and Lemma 2 applies. Therefore mean demand is $\mathrm{C}^{1}$ at $p^{\circ}$, although all preferences considered in this example exhibit a multiple jump.

\subsection{Critical jump: Fold}

Imagine the preference pattern on $X \subset \mathbb{R}^{2}$ as depicted in fig 4 . To each indifference curve there belongs a jump. The lowest and the highest budget line in the drawing, $a^{\prime \prime}$ and $c^{\prime \prime}$, are parallel. For any other budget line in between $a^{\prime \prime}$ and $c^{\prime \prime}$ and parallel to $a^{\prime \prime}$ and $c^{\prime \prime}$, demand is on the left. For any parallel budget line below $a^{\prime \prime}$ or above $c^{\prime \prime}$, demand is on the right. A similar behavior occurs if we consider families of flatter parallel budget lines until we reach the slope of the budget line $b^{\prime \prime}$ through commodity bundles $x$ and $y$. The budget line through $x$ and $y$ is the 'flattest' associated with a jump. It is characterized by the fact that $\mathrm{D} u(x)=\mathrm{D} u(y)$. Fig. 5 represents the set $i\left(\Gamma_{\lesssim}\right)$ of budget situations at which a jump occurs in the price-wealth space. The price system associated with budget lines $a^{\prime \prime}$ and $c^{\prime \prime}$ is a regular value of the projection $\pi: i\left(\Gamma_{\S}\right) \rightarrow S$. The price system associated with budget line $b^{\prime \prime}$ is a critical value of the same projection. Integrating demand with respect to wealth, given $\lesssim$, at $\bar{p}$, one obtains three integrals of $\mathrm{C}^{1}$ functions, the boundaries of integration being given by $i\left(\Gamma_{\precsim}\right)$. Hence mean demand, given $\precsim$, i.e., $\Phi(\precsim, \cdot): S \rightarrow \mathbb{R}^{l}$ is $C^{1}$ at $\bar{p}$ [cf. Dierker et al. (1980b)]. When we approach $p^{0}$ from the left, the slope of $\left.\Phi(\lesssim,)^{\cdot}\right)$ will not stay bounded as the

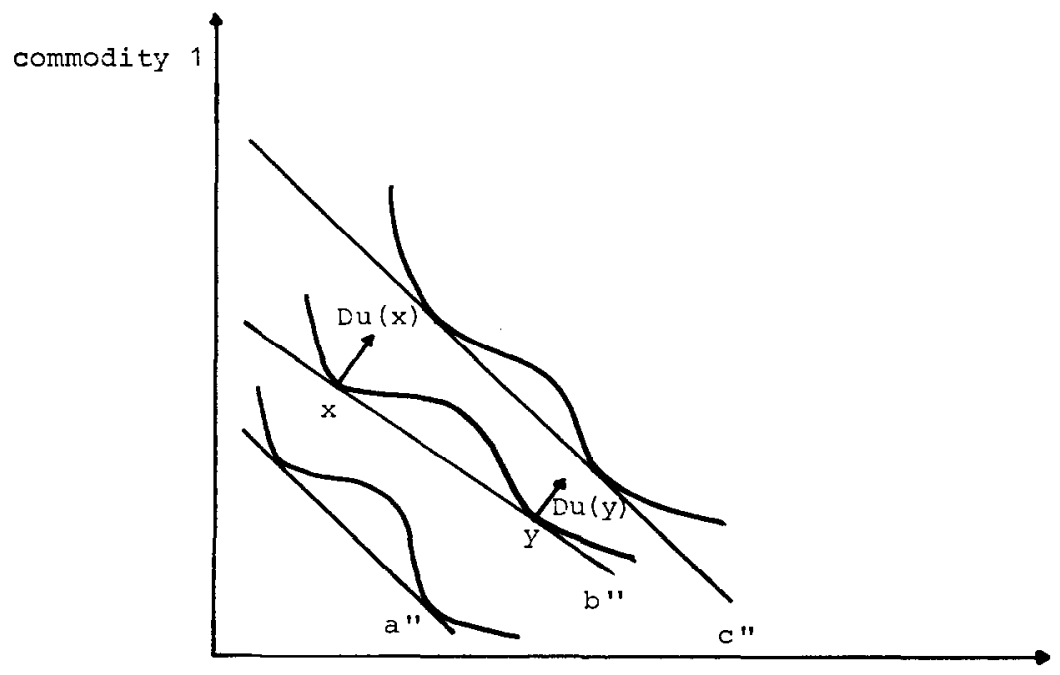

Fig. 4 


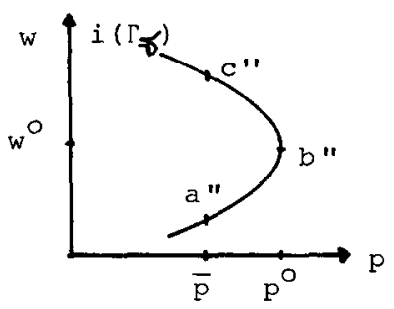

Fig. 5

boundaries of integration become arbitrarily steep. Therefore $\Phi(\lesssim, \cdot)$ is not $\mathrm{C}^{1}$ at $p^{0}$.

Now we vary the preference by stretching the vertical axis of the positive orthant and proceed as in the first example. Putting $\log p$ instead of $p$ on the horizontal axis of the diagram (fig. 5), we see that the orbit of $\lesssim$ corresponds to a family of transformed folds differing from each other by a horizontal translation. The integrability of the Lipschitz constants, called $j(\precsim)$ in Lemma 2, does not depend on the rescaling $p \mapsto \log p$ of the horizontal axis. To estimate the magnitudes of the Lipschitz constants we observe that differences in demand aggregated with respect to wealth are essentially determined by the shape of $i\left(\Gamma_{\Sigma}\right)$. Assumption $\left(\mathscr{M}_{\text {Morin }}\right)$ says that $i\left(\Gamma_{\S}\right)$ looks like a parabola $w=\sqrt{p}$ in a neighborhood $U$ of $\left(p^{0}, w^{0}\right)$. Then $i\left(\Gamma_{\Im_{q}}\right)$ is obtained by shifting $i\left(\Gamma_{\lesssim}\right)$ by $q$. Elementary geometrical considerations show that the Lipschitz constant associated with $\precsim_{q}$ is of the order $|q|^{-\frac{1}{2}}$, hence integrable with respect to the uniform distribution on $\bar{B}$.

\subsection{Critical jump: Cusp}

We assume now that we have three commodities and a preference ordering with a critical jump $\left(p^{0}, x, y\right)$ occuring at budget $\left(p^{0}, w^{0}\right)$. The assumption ( $\left.\mathscr{M}_{\text {Morin }}\right)$ says that $i\left(\Gamma_{\precsim}\right)$ exhibits either a jump or a fold at $\left(p^{0}, w^{0}\right)$. Suppose it is a cusp. The orbit of $\lesssim$ consists of preferences which also exhibit cusps. Taking $\log p_{1}$ and $\log p_{2}$ as coordinates for the price space, these cusps appear as translations in the price-wealth space of the cusp associated with $\precsim$. These translations keep wealth $w$ fixed. The Lipschitz constant $j\left(\lesssim_{q}\right)$ of Lemma 2 is essentially determined by the geometrical shape of $i\left(\Gamma_{\Im_{q}}\right)$ and by the position of the center of the cusp associated with $\precsim_{q}$. Since the cusps under consideration are all the same up to translation, we map each of them to a standard cusp centered at the origin in $\mathbb{R}^{2} \times \mathbb{R}$ and look at the image of $p^{0}$ under this mapping. As the original cusps are distributed with a bounded density with respect to Lebesgue measure, restricted to a compact $\bar{B} \subset S$, the images of $p^{0}$ under the mappings to the standard cusp are distributed with a bounded density with respect to Lebesgue measure on some compact cube $C$ centered at 0 . 
Instead of estimating the Lipschitz constant of a preference with respect to $p^{0}$, we shall estimate the Lipschitz constant of a point (the image of $p^{0}$ under the mapping to the standard cusp) with respect to the standard cusp.

The equation for the standard cusp is

$$
p_{1}+p_{2} w+w^{3}=0
$$

The standard cusp enjoys the remarkable property that the change of coordinates $p_{1} \mapsto \alpha^{3} p_{1}, p_{2} \mapsto \alpha^{2} p_{2}, w \mapsto \alpha w, \alpha>0$, leaves the surface $p_{1}+p_{2} w$ $+w^{3}=0$ invariant. Estimating the Lipschitz constant of a point $\left(p_{1}, p_{2}\right)$ thus gives an estimate for the Lipschitz constant of any point $\left(\alpha^{3} p_{1}, \alpha^{2} p_{2}\right), \alpha>0$. The Lipschitz constant of a point relates differences in direction of the $w$-axis to differences in the $\left(p_{1}, p_{2}\right)$-plane. Going from $\left(p_{1}, p_{2}\right)$ to $\left(\alpha^{3} p_{1}, \alpha^{2} p_{2}\right)$, $0<\alpha<1$, lets the Lipschitz constant increase by no more than $(1 / \alpha)^{2}$.

Now consider the transformations $T: \mathbb{R}^{2} \times \mathbb{R} \rightarrow \mathbb{R}^{2} \times \mathbb{R}$ defined by

$$
T\left(p_{1}, p_{2}, w\right)=\left(\left(\frac{1}{2}\right)^{3} p_{1},\left(\frac{1}{2}\right)^{2} p_{2}, \frac{1}{2} w\right)
$$

and

$$
\hat{T}: \mathbb{R}^{2} \rightarrow \mathbb{R}^{2} \quad \text { defined by } \quad \hat{T}\left(p_{1}, p_{2}\right)=\left(\left(\frac{1}{2}\right)^{3} p_{1},\left(\frac{1}{2}\right)^{2} p_{2}\right) .
$$

Let $C$ denote a compact cube in $\mathbb{R}^{2}$, centered at the origin and parallel to the axes. Let $C^{0}=C \backslash \hat{T} C, C^{1}=\hat{T}\left(C^{0}\right)$, etc. Then $C \backslash \bigcup_{i=0}^{\infty} C^{i}$ is a null set. Instead of integrating the Lipschitz constants over $C$, we integrate over $\bigcup_{i=0}^{\infty} C^{i}$.

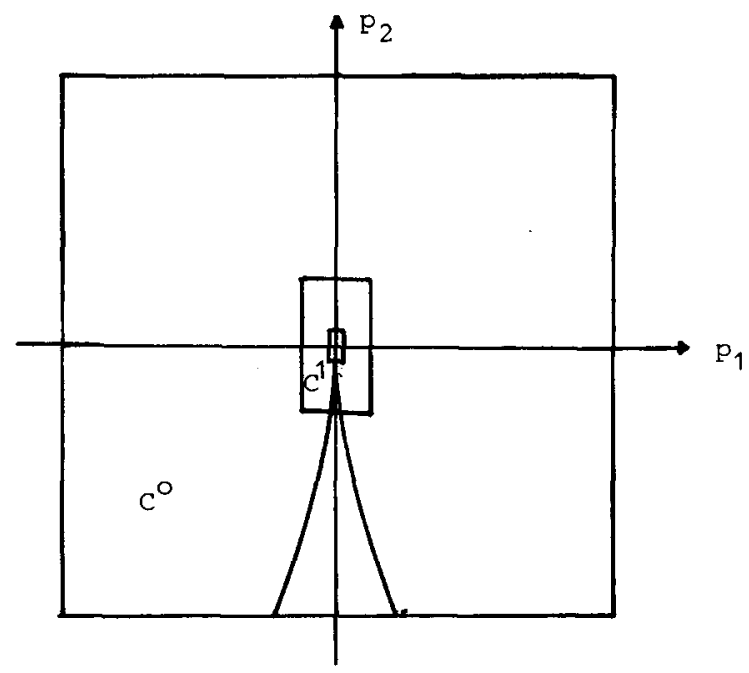

Fig. 6 
The set $C^{0}$ is bounded away from the origin. As we have seen in the previous example that the occurrence of folds does not destroy the integrability of the Lipschitz constants, it is plausible that the Lebesgue integral of the Lipschitz constants over $C^{0}$ exists. Applying transformation $\hat{T}$ to $C^{0}$ leads to an increase of the Lipschitz constants by the order $2^{2}$ and to a change of the size of an infinitesimal rectangle in $C^{0}$ by the order of $\left(\frac{1}{2}\right)^{2}\left(\frac{1}{2}\right)^{3}$ $=\left(\frac{1}{2}\right)^{5}$. Therefore the integral of the Lipschitz constants over the cube $C$ which equals the sum over the corresponding integrals over all cubes $C^{i}$, exists as $\sum_{i=0}^{\infty}\left(\frac{1}{2}\right)^{3 i}$ is finite.

In this example we have exploited a particular invariance property of the cusp. The advantage of this approach as compared to more direct estimations of the integrals of Lipschitz constants as in the fold example is mainly that it can easily be extended to the swallow-tail, then to the butterfly, and, by induction, to any 'generalized cusp' as we have completely similar invariance properties in all these cases.

\section{Proofs}

\subsection{Proof of Lemma 1}

Let $X_{1}=\left\{x \in X \mid\right.$ Hessian of $\left.u\right|_{\mathrm{KerD} u(x)}$ is negative definite $\}$. Define $\left.d: X_{1} \rightarrow S \times\right] 0, \infty\left[\right.$ by $d(x)=(g(x), g(x) x)$. Then $\operatorname{rank}(d)=l$ everywhere on $X_{1}$ [cf. Debreu (1972)]. Let $(\bar{p}, \bar{x}, \bar{y}) \in \Gamma_{\Im}$. Since $\bar{x}$ and $\bar{y}$ are in $X_{1}$, there are open neighborhoods $U(\bar{x})$ of $\bar{x}$ in $X_{1}, U(\bar{y})$ of $\bar{y}$ in $X_{1}$, and $V$ of $(\bar{p}, \bar{p} \bar{x})=(\bar{p}, \bar{p} \bar{y})$ in $S \times] 0, \infty\left[\right.$ such that $U(\bar{x}) \cap U(\bar{y})=\emptyset$ and $\left.d\right|_{U(\bar{x})}$ and $\left.d\right|_{U(\bar{y})}$ are diffeomorphisms onto $V$. Consider the mapping $V \rightarrow S \times U(\bar{x}) \times U(\bar{y}) \subset S \times \mathbb{R}^{l} \times \mathbb{R}^{l}$, defined by $(p, w) \mapsto\left(p,\left(\left.d\right|_{U(\bar{x})}\right)^{-1}(p, w),\left(\left.d\right|_{U(\bar{y})}\right)^{-1}(p, w)\right)$. It is a diffeomorphism onto its image, which is an $l$-dimensional manifold containing $\Gamma_{\lessgtr} \cap(S \times U(\bar{x}) \times U(\bar{y}))$ as a hypersurface. The restriction of this diffeomorphism to the pre-image of $\Gamma_{\Im} \cap(S \times U(\bar{x}) \times U(\bar{y}))$ has $i$ as a left inverse. Therefore the rank of $i$ at $(\bar{p}, \bar{x}, \bar{y})$ is $l-1$. Q.E.D.

\subsection{Proof of the Theorem}

Note that

$$
\begin{aligned}
\Phi(\precsim, \cdot) & =\int_{0}^{\infty} \phi(\precsim, ;, w) \mathrm{d} \delta_{\lessgtr}(w), \\
& =\int_{0}^{\infty} \phi(\precsim, \cdot, w) h_{\precsim}(w) \mathrm{d} w \quad \text { where } \quad \precsim \in \operatorname{supp} \mu,
\end{aligned}
$$

is a family of (single-valued) functions, because of $\left(\mathscr{M}_{w}\right)$ and $\left(\mathscr{M}_{\text {Morin }}\right)$. 
Let $p^{0} \in S$. To check that $F(\cdot)=\int_{\mathscr{g}} \Phi(\precsim, \cdot) \mathrm{d} \mu$ is $C^{1}$ at $p^{0}$, we apply Lemma 2 to the family $\Phi(\precsim, \cdot), \precsim \in \operatorname{supp} \mu$. Lemma 2 requires $\Phi(\precsim, \cdot)$ to be $C^{1}$ at $p^{0}$ for $\mu$-almost every $\precsim \in \mathscr{P}$. From Theorem 1 in Dierker et al. (1980b) we know that for $\precsim \in \mathscr{P}$ there exists a closed null set $N_{\lessgtr}$ in the price space $S$ such that the restriction to $S \backslash N_{\lessgtr}$ of $\Phi(\lesssim, \cdot)$ is a $C^{1}$ function. This together with assumption $\left(\mathscr{M}_{p}\right)$ underlies the proof of Lemma 3.

Lemma 3. Under the assumptions of the theorem for $\mu$-almost every $\precsim \in \mathscr{P}$ mean demand $\Phi(\lesssim, \cdot)$ is $\mathrm{C}^{1}$ at $p^{0}$.

Proof. Let $\chi^{l-1}$ denote a Haar measure on $S$. By Theorem 1 in Dierker et al. (1980b) we have

$$
\chi^{l-1}\left\{q \in S \mid \Phi(\lesssim, \cdot) \text { not } C^{1} \text { at } q\right\}=0 .
$$

By the invariance of the Haar measure $\chi^{l-1}$ this is equivalent to

$$
\chi^{l-1}\left\{q \in S \mid \Phi(\lesssim, \cdot) \text { not } C^{1} \text { at } p^{0} \circ q\right\}=0 .
$$

Therefore, by $\left(\mathscr{M}_{p}\right)$, we have for all $\precsim \in \operatorname{supp}(\mu)$,

$$
\begin{aligned}
& \xi_{\lessgtr}^{\prime}\left\{q \in S \mid \Phi(\precsim, \cdot) \text { is } C^{1} \text { at } p^{0} \circ q\right\} \\
& \quad=\xi_{\precsim}^{\prime}\left\{q \in S \mid \Phi\left(\lesssim_{q-1}, \cdot\right) \text { is } C^{1} \text { at } p^{0}\right\}=1 .
\end{aligned}
$$

The identification of the measure on $S$ and on $a^{-1}(\lesssim)$ allows us to write this as

$$
\begin{aligned}
& 1=\xi_{\S}^{\prime}\left\{\left(\precsim_{q-1}, q\right) \mid \Phi\left(\precsim_{q-1}, \cdot\right) \text { is } \mathrm{C}^{1} \text { at } p^{0}\right\} \\
& =\xi_{\lessgtr}^{\prime}\left\{\left(\precsim^{\prime}, q\right) \in a^{-1}(\precsim) \mid \Phi\left(\precsim^{\prime}, \cdot\right) \text { is } \mathrm{C}^{1} \text { at } p^{0}\right\} \\
& =\xi_{\precsim}^{\prime}\left(\left.\operatorname{pr}_{\mathscr{P}}\right|_{a^{-1}(\lesssim)}\right)^{-1}\left\{\left(\lesssim^{\prime} \in \mathscr{P} \mid \Phi\left(\lesssim^{\prime}, \cdot\right) \text { is } \mathrm{C}^{1} \text { at } p^{0}\right\}\right. \text {. } \\
& =\xi_{\S}\left\{\left(\lesssim^{\prime} \in \mathscr{P}\right) \mid \Phi\left(\lesssim^{\prime}, \cdot\right) \text { is } C^{1} \text { at } p^{0}\right\} \text {. }
\end{aligned}
$$

This implies

$$
\begin{aligned}
1 & =\mu^{\prime}(\mathscr{P})=\int_{\mathscr{P}} 1 \mathrm{~d} \mu^{\prime}(\precsim)=\int_{\mathscr{P}} \xi_{\precsim}\left\{\precsim^{\prime} \in \mathscr{P} \mid \Phi\left(\precsim^{\prime}, \cdot\right) \text { is } \mathrm{C}^{1} \text { at } p^{0}\right\} \mathrm{d} \mu^{\prime}(\precsim) \\
& =\mu\left\{\precsim^{\prime} \in \mathscr{P} \mid \Phi\left(\precsim^{\prime}, \cdot\right) \text { is } \mathrm{C}^{1} \text { at } p^{0}\right\} . \quad \text { Q.E.D. }
\end{aligned}
$$

Note that we used only price-dispersion of $\mu$ in the proof rather than the stronger uniform, local price-dispersion. 
We want to find Lipschitz constants $j(\precsim)$ for $\Phi(\precsim, \cdot)$ in a neighborhood $U\left(p^{0}\right)$ of $p^{0}$ such that $j: \mathscr{P} \rightarrow \mathbb{R} \cup\{\infty\}$ is integrable.

It is sufficient to define these Lipschitz constants locally. Suppose, there exists, for every $\left(\lesssim^{i}, w^{i}\right) \in \operatorname{supp} \tau$, a neighborhood $V\left(\lesssim^{i}\right)$ of $\nwarrow^{i}$, a neighborhood $I\left(w^{i}\right)$ of $w^{i}$, a neighborhood $U_{i}\left(p^{0}\right)$ of $p^{0}$, and a measurable function

$$
L_{i}: V\left(\lesssim^{i}\right) \rightarrow \mathbb{R} \cup\{\infty\},
$$

such that

$$
\left\|\int_{I\left(w^{i}\right)} \phi(\precsim, p, w) h_{\lessgtr}(w) \mathrm{d} w-\int_{I(w i)} \phi\left(\lesssim, p^{0}, w\right) h_{\S}(w) \mathrm{d} w\right\| \leqq L_{i}(\precsim)\left\|p-p^{0}\right\|
$$

for all $\lesssim \in V\left(\lesssim^{i}\right) \cap \operatorname{supp} \mu$ and $p \in U_{i}\left(p^{0}\right)$,

(ii) $\int_{V\left(\lesssim^{i}\right)} L_{i}(\lesssim) \mathrm{d} \mu<\infty$.

Since $\operatorname{supp} \tau$ is compact, there are finitely many neighborhoods $\left\{V\left(\lesssim^{i}\right) \times I\left(w^{i}\right)\right\}_{i=1, \ldots, n}$ which cover supp $\tau$. It is easy to check that $j(\lesssim)=$ $\sum_{\left\{i \mid \Im \in V\left(\S_{i}\right)\right\}} L_{i}(\lesssim)$ is an integrable Lipschitz constant for $\Phi(\lesssim, \cdot)$ in the neighborhood $U\left(p^{0}\right)=\bigcap_{i=1}^{n} U_{i}\left(p^{0}\right)$ of $p^{0}$.

In order to define the local Lipschitz constants, we distinguish three cases.

Case 1. Individual demand is single-valued. Let $\left(\lesssim^{0}, w^{0}\right) \in \operatorname{supp} \tau$ and assume that $\phi\left(\precsim^{0}, p^{0}, w^{0}\right)=\left\{x^{0}\right\}$. Since $\left.\phi: \mathscr{P} \times S \times\right] 0, \infty[\rightarrow X$ is u.h.c., there exist, for $\varepsilon>0$, a neighborhood $V_{1}\left(\nwarrow^{0}\right)$ of $\precsim^{0}$, a neighborhood $U_{1}\left(p^{0}\right)$ of $p^{0}$ and a neighborhood $I_{1}\left(w^{0}\right)$ of $w^{0}$ such that $\phi(\lesssim, p, w)$ is contained in the $\varepsilon$ neighborhood $U_{\varepsilon}\left(x^{0}\right)$ of $x^{0}$, if $(\precsim, p, w) \in V_{1}\left(\lesssim^{0}\right) \times U_{1}\left(p^{0}\right) \times I_{1}\left(w^{0}\right)$. Because of $\left(\mathscr{M}_{\text {curv }}\right)$ the Gaussian curvature $\kappa\left(\lesssim^{0}, x^{0}\right)$ of the $\precsim^{0}$-hypersurface through $x^{0}$ is not zero at $x^{0}$.

Claim. There are neighborhoods $V_{2}\left(\nwarrow^{0}\right)$ of $\nwarrow^{0}$ in $V_{1}\left(\nwarrow^{0}\right), U_{2}\left(p^{0}\right)$ of $p^{0}$ in $U_{1}\left(p^{0}\right)$ and a neighborhood $I_{2}\left(w^{0}\right) \subset I_{1}\left(w^{0}\right)$ of $w^{0}$ such that $\phi(\lesssim, p, w)$ is single-valued if $(\lesssim, p, w) \in V_{2}\left(\nwarrow^{0}\right) \times U_{2}\left(p^{0}\right) \times I_{2}\left(w^{0}\right)$.

Proof of the claim. Assume the claim is false. Then there are sequences $\left(\Xi^{n}\right) \rightarrow \lesssim^{0}, \quad\left(p^{n}\right) \rightarrow p^{0}, \quad\left(w^{n}\right) \rightarrow w^{0}, \quad\left(x^{n}, y^{n}\right) \in U_{\varepsilon}\left(x^{0}\right) \times U_{\varepsilon}\left(x^{0}\right) \quad$ such that $\left(x^{n}, y^{n}\right) \in \phi\left(\lesssim^{n}, p^{n}, w^{n}\right)$ and $x^{n} \neq y^{n}$. We can assume that $\left(x^{n}\right) \rightarrow x^{0},\left(y^{n}\right) \rightarrow x^{0}$. Then $\kappa\left(\Im^{0}, x^{0}\right)=0$ contrary to the assumption. $\quad$ Q.E.D.

Consequently, $\phi: V_{2}\left(\nwarrow^{0}\right) \times U_{2}\left(p^{0}\right) \times I_{2}\left(w^{0}\right) \rightarrow \mathbb{R}^{l}$ is a continuous function, and for $\precsim \in V_{2}\left(\precsim^{0}\right)$ the function $\phi(\lesssim, \cdot, \cdot)$ is $C^{1}$ at $(p, w)$ because $\kappa(\precsim, x) \neq 0$. The derivative $\mathrm{D}_{p} \phi(\precsim, p, w)$ is jointly continuous in $(\precsim, p, w)$.

Thus there are neighborhoods $V\left(\lesssim^{0}\right)$ of $\precsim^{0}, U\left(p^{0}\right)$ of $p^{0}$, and $I\left(w^{0}\right)$ of $w^{0}$, and a constant $L$, such that 
(i) $\quad\left\|\int_{I\left(w^{0}\right)} \phi(\lesssim, p, w) h_{\precsim}(w) \mathrm{d} w-\int_{I\left(w^{0}\right)} \phi\left(\lesssim, p^{0}, w\right) h_{\S}(w) \mathrm{d} w\right\|$

$\leqq L \cdot\left\|p-p^{0}\right\| \quad$ if $\lesssim \in V\left(\lesssim^{0}\right)$ and $p \in U\left(p^{0}\right)$.

Since $L$ is independent of $\precsim \in V\left(\precsim^{0}\right)$ :

(ii) $\int_{V(\lesssim 0)} L \mathrm{~d} \mu<\infty$.

When individual demand $\phi\left(\nwarrow^{0}, p^{0}, w^{0}\right)$ is not single-valued, ( $\left.\mathscr{M}_{\text {curv }}\right)$ and ( $\mathscr{U} .2)$ imply that $\phi\left(\lesssim^{0}, p^{0}, w^{0}\right)$ consists of finitely many points. There occur two cases:

(a) there is no $\left(p^{0}, x, y\right) \in \Gamma_{\Im 0}$ which is a critical point of $\pi \circ i: \Gamma_{\lessgtr 0} \rightarrow S$ and fulfills $p^{0} x=p^{0} y=w^{0}$,

(b) there exists $\left(p^{0}, x, y\right) \in \Gamma_{\preccurlyeq 0}$ which is a critical point of $\pi \circ i: \Gamma_{\preccurlyeq 0} \rightarrow S$ and fulfills $p^{\circ} x=p^{n} y=w^{0}$.

Case 2. Non-critical jumps. Let $\left(\precsim^{0} \cdot w^{0}\right) \in \operatorname{supp} \tau$ and assume that no point $\left(p^{0}, x, y\right) \in \Gamma_{\lessgtr 0}$ such that $p^{0} x=p^{0} y=w^{0}$ is a critical point of $\pi \circ i: \Gamma_{\lessgtr 0} \rightarrow S$. In the notation of Dierker et al. (1980b) this implies that rank $D_{(x, y)} G_{1}\left(p^{0}, x, y\right)$ $=2 l$ for $\left(p^{0}, x, y\right) \in \Gamma_{\precsim 0}$ such that $p^{0} x=p^{0} y=w^{0}$. Here we need a slightly different characterization of jumps.

Consider the mapping

$$
G: \hat{\mathscr{P}} \times S \times(X \times X \backslash \text { diagonal }) \rightarrow \mathbb{R}^{l-1} \times \mathbb{R} \times \mathbb{R}^{l-1} \times \mathbb{R},
$$

defined by

$$
G(u, p, x, y)=\left(\hat{g}_{\precsim}(x)-\hat{p}, u(x)-u(y), \hat{g}_{\precsim}(y)-\hat{p}, p(x-y)\right),
$$

where $u$ is a utility function for $\precsim \in \mathscr{P}$, and where $\hat{z}$ denotes the first $l-1$ components of a vector $z$ in $\mathbb{R}^{l}$. Clearly, $G$ is jointly continuous in all variables and $C^{\infty}$ with respect to $(p, x, y)$. The derivative $\mathrm{D}_{(p, x, y)} G$ is jointly continuous in $(u, p, x, y)$.

If the Hessians of $u_{\mid \operatorname{Ker} D u(x)}$ and of $u_{\mid \operatorname{Ker} \mathrm{D} u(y)}$ are negative definite, the argument in Dierker et al. (1980b) shows that

$$
\operatorname{rank} \mathrm{D}_{(p, x, y)} G(u, p, x, y)=2 l \text {. }
$$

Let $u^{0}$ be a utility for $\lesssim^{0}$. For a non-critical jump $\left(p^{0}, x, y\right) \in \Gamma_{\precsim 0}, p^{0} x=$ $p^{0} y=w^{0}$, we even have

$$
\operatorname{rank} \mathrm{D}_{(x, y)} G\left(u^{0}, p^{0}, x, y\right)=2 l .
$$

Then the implicit function theorem [cf. Schwartz (1967, p. 278)] implies that 
there are neighborhoods $V\left(u^{0}\right)$ of $u^{0}$ in $\hat{P}, U\left(p^{0}\right)$ of $p^{0}$ in $S$ and $W(x, y)$ in $X \times X \backslash$ diagonal and a pair of continuous functions

$$
(\tilde{\tilde{x}}, \tilde{y}): V\left(u^{0}\right) \times U\left(p^{0}\right) \rightarrow W(x, y)
$$

such that $G(u, p, \tilde{x}(u, p), \tilde{y}(u, p))=0$. It is easy to show that $\mathrm{D}_{p} \tilde{x}(u, p)$ and $\mathrm{D}_{p} \tilde{\tilde{y}}(u, p)$ are jointly continuous in $(u, p)$. Therefore

$$
\tilde{\tilde{w}}: V\left(u^{0}\right) \times U\left(p^{0}\right) \rightarrow \mathbb{R}:(u, p) \mapsto p \cdot \tilde{\tilde{x}}(u, p)=p \cdot \tilde{\tilde{y}}(u, p)
$$

has a derivative with respect to $p$ which is jointly continuous in $(u, p)$.

Since the projection $\widehat{\mathscr{P}}_{\rightarrow} \rightarrow \mathscr{P}$ is an open mapping, the implicit function theorem also entails the existence of a neighborhood $V\left(\lesssim^{0}\right)$ of $\lesssim^{0}$ and of continuous functions

$$
\begin{aligned}
& \tilde{x}(\cdot, \cdot): V\left(\nwarrow^{0}\right) \times U\left(p^{0}\right) \rightarrow W(x, y), \\
& \tilde{y}(\cdot, \cdot): V\left(\precsim^{0}\right) \times U\left(p^{0}\right) \rightarrow W(x, y),
\end{aligned}
$$

such that

$$
(p, \tilde{x}(\precsim, p), \tilde{y}(\precsim, p)) \in \Gamma_{\precsim}
$$

and

$$
\left(p^{0}, \tilde{x}\left(\lesssim^{0}, p^{0}\right), \tilde{y}\left(\lesssim^{0}, p^{0}\right)\right)=\left(p^{0}, x, y\right)
$$

Moreover, $\mathrm{D}_{p} \tilde{x}(\precsim, p)$ and $\mathrm{D}_{p} \tilde{y}(\precsim, p)$ exist and are jointly continuous in (,$p$ ). Also we can assume that for $\precsim \in V\left(\precsim^{0}\right)$ the set

$$
\left\{p, \tilde{x}(\precsim, p), \tilde{y}(\precsim, p) \mid p \in U\left(p^{0}\right)\right\}
$$

contains only regular points of $\mathrm{pr}_{s}: \Gamma_{\lessgtr} \rightarrow S$.

Now we continue our treatment of non-critical jumps. Observe that

$$
\left.\left\{p^{0}, x, y\right) \in \Gamma_{\preccurlyeq 0} \mid p^{0} x=p^{0} y=w^{0}\right\}
$$

contains a finite number of points $\left(p^{0}, x^{i}, y^{i}\right), i=1, \ldots, r$ to each of which we apply the procedure just described. For $i=1, \ldots, r$, let $\tilde{x}^{i}(\precsim, p), \tilde{y}^{i}(\precsim, p)$ be defined in a neighborhood $V_{i}\left(\precsim^{0}\right) \times U_{i}\left(p^{0}\right)$, by the implicit function theorem.

Claim. There exist neighborhoods $V\left(\precsim^{0}\right)$ of $\lesssim^{0}, U\left(p^{0}\right)$ of $p^{0}$, an open interval $I\left(w^{0}\right)$ containing $w^{0}$, and a number $c>0$ such that $\phi(\precsim, p, w)$ is single-valued if $(\precsim, p, w) \in V\left(\precsim^{0}\right) \times U\left(p^{0}\right) \times I\left(w^{0}\right)$ and $\left|w-w^{0}\right| \geqq c\left\|p-p^{0}\right\|$. 
Proof. Suppose the claim is false. Then, for every $c>0$, there are sequences $\left(\lesssim^{n)} \rightarrow \lesssim^{0}, \quad\left(p^{n}\right) \rightarrow p^{0}, \quad\left(w^{n}\right) \rightarrow w^{0} \quad\right.$ with $\left|w^{n}-w^{0}\right| \geqq c\left\|p^{n}-p^{0}\right\|$, and $\left(x^{n}, y^{n}\right) \in$ $\phi\left(\lesssim^{n}, w^{n}\right)$ such that $x^{n} \neq y^{n}$. Since $\mathrm{D}_{p} \tilde{w}^{i}(\precsim, p)$ is jointly continuous in $(\precsim, p)$ there is $\delta, 0<\delta<\infty$, such that for $i=1, \ldots, r$, and $(\precsim, p) \in \bigcap_{i=1}^{r}$ $V_{i}\left(\lesssim^{0}\right) \times U_{i}\left(p^{0}\right)$

$$
\left\|\mathrm{D}_{p} \tilde{w}^{i}(\lesssim, p)-\mathrm{D}_{p} \tilde{w}^{i}\left(\lesssim^{0}, p^{0}\right)\right\|<\delta .
$$

Choose $c=\delta+\max _{i=1, \ldots, r}\left\|\mathrm{D}_{p} \tilde{w}^{i}\left(\lesssim^{0}, p^{0}\right)\right\|$.

Let $\phi\left(\lesssim^{0}, p^{0}, w^{0}\right)=\left\{\bar{x}^{1}, \ldots, \bar{x}^{m}\right\}$ and let $\varepsilon>0$ bc such that the $\varepsilon$ neighborhood $U_{\varepsilon} \phi\left(\lesssim^{0}, p^{0}, w^{0}\right)$ of $\phi\left(\lesssim^{0}, p^{0}, w^{0}\right)$ is the disjoint union of the $\varepsilon$ neighborhoods $U_{\varepsilon} \bar{x}^{i}$ of $\bar{x}^{i}, i=1, \ldots, m$. Because of the upper-hemicontinuity of $\phi$ we can assume that $\left(x^{n}, y^{n}\right) \in U_{\varepsilon} \phi\left(\lesssim^{0}, p^{0}, w^{0}\right)$, which implies that there are converging subsequences, say $\left(x^{n_{k}}\right) \rightarrow \bar{x}^{1}, \quad\left(y^{n_{k}}\right) \rightarrow \bar{y}^{1}, \quad\left(\bar{x}^{1}, \bar{y}^{1}\right) \in \phi\left(\precsim^{0}, p^{0}, w^{0}\right)$. Because of $\left(\mathscr{M}_{\text {curv }}\right)$ we have $\bar{x}^{1} \neq \bar{y}^{1}$. Therefore $\left(p^{0}, \bar{x}^{1}, \bar{y}^{1}\right) \in \Gamma_{\prec 0}$. Then by the implicit function theorem the jump $\left(p^{n_{k}}, x^{n_{k}}, y^{n_{k}}\right)$ is of the form

$$
\begin{aligned}
& x^{n_{k}}=\tilde{x}^{1}\left(\lesssim^{n_{k}}, p^{n_{k}}\right), y^{n_{k}}=\tilde{y}^{1}\left(\lesssim^{n_{k}}, p^{n_{k}}\right), \\
& w^{n_{k}}=p^{n_{k}} \cdot \tilde{x}^{1}\left(\lesssim^{n_{k}}, p^{\left.n_{k}\right)}=\tilde{w}^{1}\left(\lesssim^{n_{k}}, p^{n_{k}}\right) .\right.
\end{aligned}
$$

Clearly $\left(\left|w^{n_{k}}-w^{0}\right|\right) /\left(\left\|p^{n_{k}}-p^{0}\right\|\right)$ tends to $\left\|\mathrm{D}_{p} \tilde{w}^{1}\left(\lesssim^{0}, p^{0}\right)\right\|$ which is smaller than $c$ be definition of $c$.

But $\left|w^{n_{k}}-w^{0}\right| \geqq c|| p-p^{0} \|$ by assumption. This is a contradiction.

Q.E.D.

Let $\lesssim \in V\left(\lesssim^{0}\right)$ and $p$ in some neighborhood $U$ of $p^{0}$ such that $\operatorname{cl} U \subset U\left(p^{0}\right)$. Then

$$
\begin{aligned}
& \left\|\int_{I\left(w^{0}\right)} \phi(\lesssim, p, w) h_{\Im}(w) \mathrm{d} w-\int_{I(w 0)} \phi\left(\lesssim, p^{0}, w\right) h_{\precsim}(w) \mathrm{d} w\right\| \\
& \leqq \|_{I\left(w^{0}\right) \cap\left\{w|| w-w^{0}|\geqq c| \mid p-p^{0} \|\right\}} \phi(\precsim, p, w) h_{\S}(w) \mathrm{d} w \\
& -\underset{I\left(w^{0}\right) \cap\left\{w|| w-w^{0} \mid \geqq c\left\|p-p^{0}\right\|\right\}}{ } \phi\left(\lesssim, p^{0}, w\right) h_{\lessgtr}(w) \mathrm{d} w \| \\
& +\left\|\int_{w^{0}-c\left\|p-p^{0}\right\|}^{w^{0}+c\left\|p-p^{0}\right\|} \phi(\lesssim, p, w) h_{\precsim}(w) \mathrm{d} w-\int_{w^{0}-c\left\|p-p^{0}\right\|}^{w^{0}+c\left\|p-p^{0}\right\|} \phi\left(\lesssim, p^{0}, w\right) h_{\precsim}(w) \mathrm{d} w\right\| .
\end{aligned}
$$

Clearly, the first term is Lipschitz with Lipschitz constant $L$, independent 
of $\lesssim \in V\left(\precsim^{0}\right)$, because $\phi(\precsim, p, w)$ is a $C^{1}$ function for $\precsim \in V\left(\nwarrow^{0}\right), p \in U$, $\left|w-w^{0}\right| \geqq c\left\|p-p^{0}\right\|$.

Since $\phi(\lesssim, p, w)$ is uniformly bounded for $(\lesssim, p, w) \in V\left(\lesssim^{0}\right) \times U \times\left(I\left(w^{0}\right)\right.$ and since the densities $h_{\lessgtr}(w)$ have a uniform upper bound, there exists $K>0$ such that

$$
\begin{aligned}
& \left\|\int_{w^{0}-c\left\|p-p^{0}\right\|}^{w^{0}+c|| p-p^{0} \|} \phi(\lesssim, p, w) h_{\S}(w) \mathrm{d} w-\int_{w^{0}-c\left\|p-p^{0}\right\|}^{w^{0}+c\left\|p-p^{0}\right\|} \phi\left(\lesssim, p^{0}, w\right) h_{\S}(w) \mathrm{d} w\right\| \\
& \leqq K{ }_{w^{0}-c \mid\left\|p-p^{0}\right\|}^{w^{0}+c\left\|p-p^{0}\right\|} \mathrm{d} w \leqq 2 K \cdot c\left\|p-p^{0}\right\| .
\end{aligned}
$$

Thus we have shown the existence of a local integrable Lipschitz constant for the case of non-critical jumps.

Remark. Until now we only used price-dispersion and compactness of the support of $\mu$, instead of uniform, local price-dispersion. The uniform, local price-dispersion comes into play in the next step.

Case 3. Critical jumps. We first list some terminology which we use throughout the remaining part of the paper.

The manifold $\Sigma^{k} \subset \mathbb{R}^{l}$ is defined by

$$
\Sigma^{k}=\left\{\left(x_{1}, \ldots, x_{l}\right) \in \mathbb{R}^{l} \mid \sum_{i=1}^{k} x_{i} x_{l}^{i-1}+x_{l}^{k+1}=0\right\}
$$

where $k \in\{0,1, \ldots, l-1\}$. In particular, $\Sigma^{0}=\left\{\left(x_{1}, \ldots, x_{l}\right) \in \mathbb{R}^{l} \mid x_{l}=0\right\}$.

Let $k \in\{0, \ldots, l-1\}$. The hypersurface $\Sigma^{k}$ divides $\mathbb{R}^{l}$ into two connected components, $G^{1}$ and $G^{2}$. More precisely, $\mathbb{R}^{l} \backslash \Sigma^{k}=G^{1} \cup G^{2}, G^{1}$ and $G^{2}$ connected. For $\hat{x} \in \mathbb{R}^{l-1}$ and $\hat{x}^{0} \in \mathbb{R}^{l-1}$, define

$$
\Delta\left(\hat{x}, \hat{x}^{0}\right)=\left\{x_{l} \in \mathbb{R}^{1} \mid\left(\hat{x}, x_{l}\right) \in G^{i},\left(\hat{x}^{0}, x_{l}\right) \in G^{j}, \text { for some } i \neq j\right\} .
$$

Observe that $\Delta\left(\hat{x}, \hat{x}^{0}\right)$ is always a bounded set. Define $Q: \mathbb{R}^{l-1} \times \mathbb{R}^{l-1}$ diagonal $\rightarrow \mathbb{R}$ by

$$
Q\left(\hat{x}, \hat{x}^{0}\right)=\left\|\hat{x}-\hat{x}^{0}\right\|^{-1} \lambda^{1}\left(\Delta\left(\hat{x}, \hat{x}^{0}\right)\right) .
$$

$H: \mathbb{R}^{l-1} \times \mathbb{R} \rightarrow \mathbb{R}^{l-1} \times \mathbb{R}$ is assumed to be a fibrewise diffeomorphism into $\mathbb{R}^{l-1} \times \mathbb{R}$. Fibrewise means that $H$ is of the form

$$
H\left(\hat{x}, x_{l}\right)=\left(H^{1}(\hat{x}), H^{2}\left(\hat{x}, x_{l}\right)\right)=\left(\hat{y}, y_{l}\right)
$$


Let $\hat{y}=H^{1}(\hat{x}), \hat{y}^{0}=H^{1}\left(\hat{x}^{0}\right)$, and $k \in\{0, \ldots, l-1\}$. Define

$$
\Delta^{H}\left(\hat{y}, \hat{y}^{0}\right)=\left\{y_{l} \in \mathbb{R}^{1} \mid\left(\hat{y}, y_{l}\right) \in H\left(G^{i}\right),\left(\hat{y}^{0}, y_{l}\right) \in H\left(G^{j}\right) \text { for some } i \neq j\right\} .
$$

For $\hat{y} \neq \hat{y}^{0}$, define

$$
Q^{H}\left(\hat{y}, \hat{y}^{0}\right)=\left\|\hat{y}-\hat{y}^{0}\right\|^{-1} \lambda^{1}\left(\Delta^{H}\left(\hat{y}, \hat{y}^{0}\right)\right) .
$$

Lemma 4. Let $\mathscr{H}$ be a set of fibrewise diffeomorphisms mapping $\mathbb{R}^{l-1} \times \mathbb{R}$ into $\mathbb{R}^{l-1} \times \mathbb{R}$. Assume $\mathscr{H}$ is compact in the topology of uniform $\mathrm{C}^{1}$-convergence on compact sets. Let $\mathrm{C} \subset \mathbb{R}^{l-1}$ be compact. Then there are constants $\gamma_{1}, \gamma_{2}$ such that the following inequality holds uniformly for $H \in \mathscr{H},\left(\hat{x}, \hat{x}^{0}\right) \in C \times C \backslash$ diagonal:

$$
Q^{H}\left(\hat{y}, \hat{y}^{0}\right) \leqq \gamma^{1}+\gamma^{2} Q\left(\hat{x}, \hat{x}^{0}\right)
$$

Proof. The set $\Delta^{H}\left(\hat{y}, \hat{y}^{0}\right)$ is composed out of two sets, because it consists of those $y_{l}$ for which $\left(\hat{y}, y_{l}\right) \in H\left(G^{1}\right)$ and of those $y_{l}$ for which $\left(\hat{y}, y_{l}\right) \in H\left(G^{2}\right)$. If one can replace $\Delta^{H}\left(\hat{y}, \hat{y}^{0}\right)$ in the definition of $Q^{H}\left(\hat{y}, \hat{y}^{0}\right)$ by any of the two constituent parts of $\Delta^{H}\left(\hat{y}, \hat{y}^{0}\right)$ and show that the statement of Lemma 4 holds with this modification, then Lemma 4 obtains. To avoid a more complex notation, we assume that one of the two constituent parts of $\Delta^{H}\left(\hat{y}, \hat{y}^{0}\right)$ is empty so that $\left(\hat{y}, y_{l}\right) \in H\left(G^{1}\right)$ and $\left(\hat{y}^{0}, y_{l}\right) \in H\left(G^{2}\right)$ for all $y_{l} \in \Delta^{H}\left(\hat{y}, \hat{y}^{0}\right)$.

Next, observe that

$$
\bigcup_{H \in \mathscr{C}}\left\{H\left(\Sigma^{k}\right) \cap\left(H^{1}(C) \times \mathbb{R}\right)\right\}
$$

is bounded. Hence $\left|\mathrm{D}_{l} H^{-1}\left(\hat{y}, y_{l}\right)\right|^{-1}$ and $\left|\mathrm{D}_{l} H^{-1}\left(\hat{y}^{0}, y_{l}\right)\right|^{-1}$ are uniformly bounded for $H \in \mathscr{H}, \hat{y} \in H^{1}(C), \hat{y}^{0} \in H^{1}(C), y_{l} \in \Delta^{H}\left(\hat{y}, \hat{y}^{0}\right)$. Therefore, by the change of variable formula for integrals, there is $\gamma^{\prime} \in \mathbb{R}$ independent of $H \in \mathscr{H}$, $\hat{y} \in H^{1}(C), \hat{y}^{0} \in H^{1}(C)$ such that

$$
\lambda^{1}\left(\{\hat{y}\} \times \Delta^{H}\left(\hat{y}, \hat{y}^{0}\right)\right) \leqq \gamma^{\prime} \cdot \lambda^{1}\left(H^{-1}\left(\{\hat{y}\} \times \Delta^{H}\left(\hat{y}, \hat{y}^{0}\right)\right)\right)
$$

and

$$
\lambda^{1}\left(\left\{\hat{y}^{0}\right\} \times \Delta^{H}\left(\hat{y}, \hat{y}^{0}\right)\right) \leqq \gamma^{\prime} \cdot \lambda^{1}\left(H^{-1}\left(\left\{\hat{y}^{0}\right\} \times \Delta^{H}\left(\hat{y}, \hat{y}^{0}\right)\right)\right) .
$$

This implies

$$
\begin{aligned}
2 \cdot \lambda^{1}\left(\Delta^{H}\left(\hat{y}, \hat{y}^{0}\right)\right) \leqq & \gamma^{\prime} \cdot \lambda^{1}\left(H^{-1}\left(\{\hat{y}\} \times \Delta^{H}\left(\hat{y}, \hat{y}^{0}\right)\right)\right) \\
& +\gamma^{\prime} \cdot \lambda^{1}\left(H^{-1}\left(\left\{\hat{y}^{0}\right\} \times \Delta^{H}\left(\hat{y}, \hat{y}^{0}\right)\right)\right)
\end{aligned}
$$




$$
\begin{aligned}
= & \gamma^{\prime} \cdot \lambda^{1}\left[\operatorname{proj}_{l} H^{-1}\left(\{\hat{y}\} \times \Delta^{H}\left(\hat{y}, \hat{y}^{0}\right)\right)\right. \\
& \left.\Delta \operatorname{proj}_{l} H^{-1}\left(\left\{\hat{y}^{0}\right\} \times \Delta^{H}\left(\hat{y}, \hat{y}^{0}\right)\right)\right] \\
& +2 \gamma^{\prime} \cdot \lambda^{1}\left[\operatorname{proj}_{l} H^{-1}\left(\{\hat{y}\} \times \Delta^{H}\left(\hat{y}, \hat{y}^{0}\right)\right)\right. \\
& \left.\cap \operatorname{proj}_{l} H^{-1}\left(\left\{\hat{y}^{0}\right\} \times \Delta^{H}\left(\hat{y}, \hat{y}^{0}\right)\right)\right],
\end{aligned}
$$

where $\Delta$ denotes the symmetric difference. The last inequality is of the type $\lambda^{1}(A)+\lambda^{1}(B)=\lambda^{1}[A \triangle B]+2 \lambda^{1}[A \cap B]$.

Now we examine the term of the form $\lambda^{1}[A \Delta B]$. The set $\Delta^{H}\left(\hat{y}, \hat{y}^{0}\right)$ is the union of intervals with boundary points in $\operatorname{proj}_{l}\left(H\left(\Sigma^{k}\right) \cap(\hat{y} \times \mathbb{R})\right)$ or in $\operatorname{proj}_{l}\left(H\left(\Sigma^{k}\right) \cap\left(\hat{y}^{0} \times \mathbb{R}\right)\right)$. Since the number of available boundary points is uniformly bounded, the number of intervals in $\Delta^{H}\left(\hat{y}, \hat{y}^{0}\right)$ is uniformly bounded. The sets $\{\hat{y}\} \times \Delta^{H}\left(\hat{y}, \hat{y}^{0}\right)$ and $\left\{\hat{y}^{0}\right\} \times \Delta^{H}\left(\hat{y}, \hat{y}^{0}\right)$ are mapped diffeomorphically by $\operatorname{proj}_{l}{ }^{\circ} H^{-1}$ into two unions of intervals, say $\cup A^{i}$ and $\cup B^{i}$. Now

$$
\cup A^{i} \triangle \cup B^{i} \subset \cup\left(A^{i} \triangle B^{i}\right)
$$

Moreover, for every $i$,

$$
\lambda^{1}\left(A^{i} \Delta B^{i}\right) \leqq 2 \cdot \sup _{y_{l} \in \Delta^{H}\left(\hat{y}, \hat{y}^{0}\right)}\left|\operatorname{proj}_{l} H^{-1}\left(\hat{y}, y_{l}\right)-\operatorname{proj}_{l} H^{-1}\left(\hat{y}^{0}, y_{l}\right)\right| .
$$

Since $\mathscr{H}$ and $C$ are compact and $\Delta^{H}\left(\hat{y}, \hat{y}^{0}\right)$ is uniformly bounded, there is $\gamma^{\prime \prime}$ such that the inequality

$$
2 \cdot \sup _{y_{l} \in \Delta^{H}\left(\hat{y}, \hat{y}^{0}\right)}\left|\operatorname{proj}_{l} H^{-1}\left(\hat{y}, y_{l}\right)-\operatorname{proj}_{l} H^{-1}\left(\hat{y}^{0}, y_{l}\right)\right| \leqq \gamma^{\prime \prime} \cdot\left\|\hat{y}-\hat{y}^{0}\right\|
$$

holds uniformly. Summarizing, there is $\gamma_{1} \in \mathbb{R}$ such that the inequality

$$
\begin{aligned}
& \frac{1}{2} \gamma^{\prime} \cdot \lambda^{1}\left[\operatorname{proj}_{l} H^{-1}\left(\{\hat{y}\} \times \Delta^{H}\left(\hat{y}, \hat{y}^{0}\right)\right) \triangle \operatorname{proj}_{l} H^{-1}\left(\left\{\hat{y}^{0}\right\} \times \Delta^{H}\left(\hat{y}, \hat{y}^{0}\right)\right)\right] \\
& \quad \leqq \gamma_{1}\left\|\hat{y}-\hat{y}^{0}\right\|
\end{aligned}
$$

holds uniformly.

Next we examine the term of the form $\lambda^{1}[A \cap B]$. Since, by assumption, $\left(\hat{y}, y_{l}\right) \in H\left(G^{1}\right)$ and $\left(\hat{y}^{0}, y_{l}\right) \in H\left(G^{2}\right)$ for all $y_{l} \in \Delta\left(\hat{y}, \hat{y}^{0}\right)$, we have

$$
\left[\operatorname{proj}_{l} H^{-1}\left(\{\hat{y}\} \times \Delta^{H}\left(\hat{y}, \hat{y}^{0}\right) \cap \operatorname{proj}_{l} H^{-1}\left(\left\{\hat{y}^{0}\right\} \times \Delta^{H}\left(\hat{y}, \hat{y}^{0}\right)\right)\right] \subset \Delta\left(\hat{x}, \hat{x}^{0}\right) .\right.
$$

Therefore

$$
\lambda^{1}\left[\operatorname{proj}_{l} H^{-1}\left(\{\hat{y}\} \times \Delta^{H}\left(\hat{y}, \hat{y}^{0}\right) \cap \operatorname{proj}_{l} H^{-1}\left(\left\{\hat{y}^{0}\right\} \times \Delta^{H}\left(\hat{y}, \hat{y}^{0}\right)\right)\right] \leqq \lambda^{1}\left(\Delta\left(\hat{x}, \hat{x}^{0}\right)\right) .\right.
$$


Furthermore, $\mathscr{H}^{1}=\left\{H^{1} \mid \exists H^{2}\right.$ such that $\left.\left(H^{1}, H^{2}\right) \in \mathscr{H}\right\}$ is compact. An application of the mean value theorem yields that there is $\gamma^{\prime \prime \prime} \in \mathbb{R}$, such that

$$
\left\|\hat{y}-\hat{y}^{0}\right\|=\left\|H^{1}(\hat{x})-H^{1}\left(\hat{x}^{0}\right)\right\| \geqq \gamma^{\prime \prime \prime}\left\|\hat{x}-\hat{x}^{0}\right\|
$$

for all $H^{1} \in \mathscr{H}^{1}, \hat{x} \in C, \hat{x}^{0} \in C$.

Finally, combining the last two paragraphs, we have

$\frac{1}{\left\|\hat{y}-\hat{y}^{0}\right\|} \cdot \gamma^{\prime} \cdot \lambda^{1}\left[\operatorname{proj}_{l} H^{-1}\left(\{\hat{y}\} \times \Delta^{H}\left(\hat{y}, \hat{y}^{0}\right)\right) \cap \operatorname{proj}_{l} H^{-1}\left(\left\{\hat{y}^{0}\right\} \times \Delta^{H}\left(\hat{y}, \hat{y}^{0}\right)\right)\right]$

$$
\leqq \frac{1}{\gamma^{\prime \prime \prime}\left\|\hat{x}-\overline{\hat{x}^{0}}\right\|} \gamma^{\gamma^{\prime}} \cdot \lambda^{1}\left(\Delta\left(\hat{x}, \hat{x}^{0}\right)\right)=\frac{\gamma^{\prime}}{\gamma^{\prime \prime \prime}} Q\left(\hat{x}, \hat{x}^{0}\right)
$$

Put $\gamma_{2}=\gamma^{\prime} / \gamma^{\prime \prime \prime}$. Q.E.D.

Lemma 5. Let $C \subset \mathbb{R}^{l-1}$ be compact, $\varepsilon>0$. Denote an $\varepsilon$-cube around $\hat{x}^{0} \in \mathbb{R}^{l-1}$ by $W_{\varepsilon}\left(\hat{x}^{0}\right)$. Assume

$$
\int_{c} \sup _{\hat{x} \in W_{e}\left(x^{0}\right)} Q\left(\hat{x}, \hat{x}^{0}\right) \lambda^{l-1}\left(\mathrm{~d} \hat{x}^{0}\right)<\infty
$$

Then

$$
\int_{C} \sup _{\hat{x} \in \mathbf{R}^{l-1}} Q\left(\hat{x}, \hat{x}^{0}\right) \lambda^{l-1}\left(\mathbf{d} \hat{x}^{0}\right)<\infty
$$

Proof. First we want to show that $Q: \mathbb{R}^{l-1} \times \mathbb{R}^{l-1} \backslash$ diagonal $\rightarrow \mathbb{R}$ is continuous.

Assume $\tilde{x}^{n} \vec{n} \hat{x}$. We claim that $\lambda^{1}\left(\Delta\left(\tilde{x}^{n}, \hat{x}\right)\right)$ tends to zero. To see this, let $\left(\hat{x}, x_{l}\right) \in G^{i}, i \in\{1,2\}$. Since $G^{i}$ is open, we have $\left(\tilde{x}^{n}, x_{l}\right) \in G^{i}$ for $n$ large enough. Let $G_{\tilde{x}^{n}}^{i}$ denote the $\tilde{x}^{n}$-section of $G^{i}$. We have $1_{G_{x^{n}}^{i}}\left(x_{l}\right) \rightarrow 1_{G_{x}^{i}}\left(x_{l}\right)$ for all $x_{l}$ and thus in $\lambda^{1}$-measure. Therefore

$$
\lambda^{1}\left(\Delta\left(\tilde{x}^{n}, \hat{x}\right)\right) \rightarrow 0 .
$$

Consider points $\tilde{x}, \tilde{x}^{0}, \hat{x}, \hat{x}^{0} \in \mathbb{R}^{l-1}$. Define

$$
\begin{aligned}
M= & \left\{x_{l} \in \mathbb{R}^{1} \mid\left(\tilde{x}, x_{l}\right) \in \Sigma^{k} \text { or }\left(\tilde{x}^{0}, x_{l}\right) \in \Sigma^{k}\right. \text { or } \\
& \left.\left(\hat{x}, x_{l}\right) \in \Sigma^{k} \text { or }\left(\hat{x}^{0}, x_{l}\right) \in \Sigma^{k}\right\} .
\end{aligned}
$$

We want to show:

$$
\Delta\left(\tilde{x}, \tilde{x}^{0}\right) \triangle \Delta\left(\hat{x}, \hat{x}^{0}\right) \subset \Delta(\tilde{x}, \hat{x}) \cup \Delta\left(\tilde{x}^{0}, \hat{x}^{0}\right) \cup M .
$$


To see this, assume $x_{l} \notin \Delta(\tilde{x}, \hat{x}) \cup \Delta\left(\tilde{x}^{0}, \hat{x}^{0}\right) \cup M$. If $x_{l} \in \Delta\left(\tilde{x}, \tilde{x}^{0}\right)$, then $x_{l} \in \Delta\left(\tilde{x}^{0}, \hat{x}\right)$ and therefore $x_{l} \in \Delta\left(\hat{x}, \hat{x}^{0}\right)$. If $x_{l} \in \Delta\left(\hat{x}, \hat{x}^{0}\right)$, then $x_{l} \in \Delta\left(\tilde{x}, \hat{x}^{0}\right)$ and therefore $x_{l} \in \Delta\left(\tilde{x}, \tilde{x}^{0}\right)$. Hence $x_{l} \notin \Delta\left(\tilde{x}, \tilde{x}^{0}\right) \Delta \Delta\left(\hat{x}, \hat{x}^{0}\right)$. The continuity of $Q$ follows from the facts that $\lambda^{1}(M)=0$ and $\lambda^{1}(\Delta(\tilde{x}, \hat{x}))+\lambda^{1}\left(\Delta\left(\tilde{x}^{0}, \hat{x}^{0}\right)\right)$ tends to zero if $\tilde{x}$ and $\hat{x}$ approach $\tilde{x}^{0}$ and $\hat{x}^{0}$ respectively.

Next we want to show that $\lim _{\|\hat{x}\| \rightarrow \infty} Q\left(\hat{x}, \hat{x}^{0}\right)=0$ uniformly in $\hat{x}^{0} \in C$. Observe, for that purpose, that $\left(\hat{x}, x_{l}\right)$ and $\left(\hat{x}^{0}, x_{l}\right)$ belong to different $G^{i}$ only if the line segment connecting them intersects $\Sigma^{k}$ in some point $\left(\hat{x}^{\prime}, x_{l}\right)=$ $\left(\hat{x}^{\prime}\left(\hat{x}, \hat{x}^{0}\right), x_{l}\right)$. We can assume $k \neq 0$. Since $\left(\hat{x}^{\prime}, x_{l}\right) \in \Sigma^{k}$ we have, for $\left|x_{l}\right| \geqq 1$,

$$
\left|x_{l}\right|^{k+1} \leqq\left|x_{1}^{\prime}\right|+\left|x_{2}^{\prime}\right|\left|x_{1}\right|+\cdots+\left|x_{k}^{\prime}\right|\left|x_{l}^{k-1}\right| \leqq k \| \hat{x}^{\prime}||\left|x_{l}\right|^{k-1} .
$$

Therefore,

$$
\left|x_{l}\right|^{2} \leqq \max \left\{1, k\left\|\hat{x}^{\prime}\right\|\right\} .
$$

For $\|\hat{x}\|$ large enough and $\hat{x}^{0} \in C, 2\left\|\hat{x}-\hat{x}^{0}\right\|>\|\hat{x}\|>\left\|\hat{x}^{\prime}\right\|$ and $\left|x_{l}\right|^{2} \leqq \max \{1, k\|\hat{x}\|\}$ $=k\|\hat{x}\|$. It follows that, for $\hat{x}^{0} \in C$,

$$
\begin{aligned}
\lim _{\|\hat{x}\| \rightarrow \infty} \frac{\lambda^{1}\left(\Delta\left(\hat{x}, \hat{x}^{0}\right)\right)}{\left\|\hat{x}-\hat{x}^{0}\right\|} & \leqq \lim _{\|\hat{x}\| \rightarrow \infty} \frac{2}{\|\hat{x}\|} \sup _{x_{l} \in \Delta\left(\hat{x}, \hat{x}^{0}\right)}\left|x_{l}\right| \\
& \leqq \lim _{\|\hat{x}\| \rightarrow \infty} \frac{2}{\|\hat{x}\|} \sqrt{k\|\hat{x}\|}=0 .
\end{aligned}
$$

Therefore $\lim _{\|x\| \rightarrow \infty} Q\left(\hat{x}, \hat{x}^{0}\right)=0$ uniformly in $\hat{x}^{0} \in C$.

Finally, it remains to show $\sup _{\hat{x}^{0} \in C} \sup _{\hat{x} \phi W_{\varepsilon}\left(\hat{x}^{0}\right)} Q\left(\hat{x}, \hat{x}^{0}\right)<\infty$. Let this be false. Then there is a sequence of points $\hat{x}^{0}$ in $\hat{C}$ and of points $\hat{x} \notin W_{\varepsilon}\left(\hat{x}^{0}\right)$ with $Q\left(\hat{x}, \hat{x}^{0}\right)$ unbounded. Since $C$ is compact and $\lim _{\|\mid \hat{x}\| \rightarrow \infty} Q\left(\hat{x}, \hat{x}^{0}\right)=0$ uniformly in $\hat{x}^{0} \in C$, we can assume that the sequence of points $\left(\hat{x}, \hat{x}^{0}\right)$ converges to a pair $\left(\bar{x}, \bar{x}^{0}\right)$ such that $\bar{x}^{0} \in C, \bar{x} \notin W_{\varepsilon}\left(\bar{x}^{0}\right)$. This contradicts the continuity of Q. Q.E.D.

Lemma 6. Let $C$ be a compact cube in $\mathbb{R}^{l-1}$ parallel to the axes and centered at the origin. Then, for $Q\left(\hat{x}, \hat{x}^{0}\right)$ defined with respect to some $\Sigma^{k}$, where $0 \leqq k \leqq l-1$,

$$
\int_{C} \sup _{\hat{x} \in \mathbb{R}^{l-1}} Q\left(\hat{x}, \hat{x}^{0}\right) \lambda^{l-1}\left(\mathrm{~d} \hat{x}^{0}\right)<\infty .
$$

Proof. The proof proceeds by induction on $k$. For $k=0, \Sigma^{k}$ equals the hyperplane defined by the equation $y_{l}=0$. Hence $Q$ is identically equal to zero. 
Assume that the lemma holds for $k^{\prime} \leqq k-1$ and consider $\Sigma^{k}$. Define $\widehat{T}: \mathbb{R}^{l-1} \rightarrow \mathbb{R}^{l-1}$ and $T: \mathbb{R}^{l} \rightarrow \mathbb{R}^{l}$ by

$$
\hat{T}\left(x_{1}, \ldots, x_{l-1}\right)=\left(\left(\frac{1}{2}\right)^{k+1} x_{1},\left(\frac{1}{2}\right)^{k} x_{2},\left(\frac{1}{2}\right)^{k-1} x_{3}, \ldots,\left(\frac{1}{2}\right)^{2} x_{k}, x_{k+1}, \ldots, x_{l-1}\right)
$$

and

$$
T\left(x_{1}, \ldots, x_{l-1}, x_{l}\right)=\left(\hat{T}\left(x_{1}, \ldots, x_{l-1}\right), \frac{1}{2} x_{l}\right)
$$

Since $C$ is parallel to the axes and centered at the origin, $\hat{T} C \subset C$. Let $C^{0}=C \backslash \hat{T} C, C^{1}=\hat{T} C^{0}, C^{2}=\hat{T} C^{1}$, etc. Then $C \backslash \bigcup_{i=0}^{\infty} C^{i}$ is a null set. We integrate over $\bigcup_{i=0}^{\infty} C^{i}$ instead of over $C$.

We can assume that $C$ has a non-empty interior. Then all singularities associated with parameter systems in $C^{0}$ have corank 1 and codimension at most equal to $k-1$. Therefore, if $\left(\hat{x}^{0}, x_{l}\right) \in \Sigma^{k}, \hat{x}^{0} \in C^{0}$, then there exist a number $k^{\prime} \leqq k-1$, a neighborhood $U$ of the origin in $\mathbb{R}^{l-1} \times \mathbb{R}$, and a fibrewise diffeomorphism mapping 0 to $\left(\hat{x}^{0}, x_{l}\right), U$ onto a neighborhood of $\left(\hat{x}^{0}, x_{l}\right)$, and $\Sigma^{k^{\prime}} \cap U$ into $\Sigma^{k}$. By the induction hypothesis and Lemmas 4 and 5 , there exists a neighborhood $C\left(\hat{x}^{0}\right)$ around $\hat{x}^{0} \in \mathbb{R}^{l-1}$, such that

$$
\int_{C\left(\hat{x}^{0}\right)} \sup _{\hat{x} \in \mathbb{R}^{l-1}} Q\left(\hat{x}, \hat{x}^{0}\right) \lambda^{l-1}\left(\mathrm{~d} \hat{x}^{0}\right)<\infty
$$

The compact set $C$ can be covered by a finite number of such neighborhoods. Hence

$$
\int_{C^{0}} \sup _{\hat{x} \in \mathbb{R}^{l-1}} Q\left(\hat{x}, \hat{x}^{0}\right) \lambda^{l-1}\left(\mathrm{~d} \hat{x}^{0}\right)=\alpha<\infty
$$

Since $\left|(\hat{T} \hat{x})_{h}\right| \geqq\left(1 / 2^{k+1}\right)\left|\hat{x}_{h}\right|$ for each component $h$ of every vector $\hat{x}$

$$
\left\|\hat{T} \hat{x}-\hat{T} \hat{x}^{0}\right\| \geqq \frac{1}{2^{k+1}}\left\|\hat{x}-\hat{x}^{0}\right\|
$$

The transformation $T$ leaves the hypersurface $\Sigma^{k}$ invariant. Hence

$$
Q\left(\hat{T} \hat{x}, \hat{T} \hat{x}^{0}\right) \leqq \frac{2^{k+1}}{\left\|\hat{x}-\hat{x}^{0}\right\|} \cdot \frac{1}{2} \lambda^{1}\left(\Delta\left(\hat{x}, \hat{x}^{0}\right)\right)=2^{k} Q\left(\hat{x}, \hat{x}^{0}\right)
$$

Therefore, for every $\hat{x}^{0}$,

$$
\sup _{\hat{x} \in \mathrm{R}^{l-1}} Q\left(\hat{T} \hat{x}, \hat{T} \hat{x}^{0}\right) \leqq 2^{k} \sup _{\hat{x} \in \mathbf{R}^{l-1}} Q\left(\hat{x}, \hat{x}^{0}\right) .
$$

The determinant of $\widehat{T}$ is $\left(\frac{1}{2}\right)^{k+1} \cdot\left(\frac{1}{2}\right)^{k} \cdot \ldots \cdot\left(\frac{1}{2}\right)^{2} \leqq\left(\frac{1}{2}\right)^{k+1}$. The change of variable formula for integrals yields 


$$
\int_{\mathbf{C}^{1}} \sup _{\hat{x} \in \mathbb{R}^{l-1}} Q\left(\hat{x}, \hat{x}^{0}\right) \lambda^{l-1}\left(\mathrm{~d} \hat{x}^{0}\right) \leqq \int_{C^{0}} \sup _{\hat{x} \in \mathbb{R}^{l-1}} 2^{k} Q\left(\hat{x}, \hat{x}^{0}\right) \cdot\left(\frac{1}{2}\right)^{k+1} \lambda^{l-1}\left(\mathrm{~d} x^{0}\right)=\frac{1}{2} \alpha .
$$

Similarly,

$$
\int_{C^{2}} \sup _{\hat{x} \in \mathbb{R}^{l-1}} Q\left(\hat{x}, \hat{x}^{0}\right) \lambda^{l-1}\left(\mathrm{~d} \hat{x}^{0}\right) \leqq\left(\frac{1}{2}\right)^{2} \alpha, \text { etc. }
$$

Therefore,

$$
\sum_{i=0}^{\infty} \int_{C^{i}} \sup _{\hat{x} \in \mathbb{R}^{i-1}} Q\left(\hat{x}, \hat{x}^{0}\right) \lambda^{l-1}\left(\mathrm{~d} \hat{x}^{0}\right) \leqq \sum_{i=0}^{\infty}\left(\frac{1}{2}\right)^{i} \alpha<\infty . \quad \text { Q.E.D. }
$$

Lemmas 4, 5 and 6 are purely mathematical tools which come into play by assumption $\left(\mathscr{M}_{\text {Morin }}\right)$. Let $\left(\nwarrow^{0}, w^{0}\right) \in \operatorname{supp} \tau$ and let $\left(p^{0}, x^{0}, y^{0}\right) \in \Gamma_{\preccurlyeq 0}$ be a critical point of $\pi \circ i: \Gamma_{\preccurlyeq 0} \rightarrow S$. By $\left(\mathscr{M}_{\text {Morin }}\right)$ there is a neighborhood $\widetilde{V}$ of $\precsim^{0}$ such that $\left.H_{\precsim}: \mathbb{R}^{l-1} \times \mathbb{R} \rightarrow S \times\right] 0, \infty$ [ is a fibrewise diffeomorphism for all $\precsim \in V$. We can assume that $V$ is chosen so small that there are neighborhoods $U^{1}$ of $p^{0}$ in $S$ and $U^{2}$ of $w^{0}=p^{0} x^{0}=p^{0} y^{0}$ in $] 0, \infty[$ such that $U^{1} \times U^{2} \subset$ image $H_{\precsim}$ for all $\lesssim \in V$. Let $\tilde{V} \subset V, \tilde{U}^{1} \subset U^{1}, \tilde{U}^{2} \subset U^{2}$ be neighborhoods of $\precsim^{0}, p^{0}, w^{0}$, respectively, such that $\tilde{V} \cap \operatorname{supp} \mu, \tilde{U}^{1}, \tilde{U}^{2}$ are compact.

In order to show the integrability of an appropriate set of local Lipschitz constants, we consider terms of the form

$$
\sup _{p \in \tilde{U}^{1}}\left\|p-p^{0}\right\|^{-1}\left\|\int_{\tilde{U}^{2}}\left(\phi(\lesssim, p, w)-\phi\left(\lesssim, p^{0}, w\right)\right) h_{\precsim}(w) \mathrm{d} w\right\|,
$$

where $\lesssim \in \tilde{V}$.

Let $\precsim \in V,(p, w) \in$ image $H_{\Im}$, and $\left(p^{o}, w\right) \in$ image $H_{\lessgtr}$. Since $\mathbb{R}^{l-1} \times \mathbb{R} \backslash \Sigma^{k}$ is composed of the open sets $G^{1}$ and $G^{2}$, demand in image $H_{\lessgtr}$ is described by two smooth functions, $\phi^{1}$ and $\phi^{2}$, which are associated with $H_{\S}\left(G^{1}\right)$ and $H_{\precsim}\left(G^{2}\right)$.

Firstly, consider the case in which $(p, w)$ belongs to $H_{\precsim}\left(G^{1}\right)$ and $\left(p^{0}, w\right)$ belongs to $H_{\precsim}\left(G^{2}\right)$, or vice versa, $\lesssim \in \tilde{V}$. Then there is a constant not depending on $\widetilde{p} \in \tilde{U}^{1}, w \in \tilde{U}^{2}$, $\in \tilde{V} \cap \operatorname{supp} \mu$ such that

$$
\left\|\phi(\precsim, p, w)-\phi\left(\precsim, p^{0}, w\right)\right\| h_{\precsim}(w) \leqq \text { constant. }
$$

Here we made use of $\left(\mathscr{M}_{w}\right)$. For the purpose of integrating local Lipschitz constants we can assume that constant $=1$ in order to simplify notation.

Secondly, consider the case in which $(p, w)$ and $\left(p^{0}, w\right)$ both belong to the same $H_{\Im}\left(G^{i}\right), i=1,2$. Then there is a constant, not depending on $p \in \tilde{U}_{1}$, $w \in \tilde{U}_{2}, \precsim \in \widetilde{V} \cap \operatorname{supp} \mu$, such that

$$
\begin{aligned}
& \left\|p-p^{0}\right\|^{-1}\left\|\phi(\precsim, p, w)-\phi\left(\precsim, p^{0}, w\right)\right\| h_{\lessgtr}(w) \\
& \quad \leqq \sup _{\substack{(p, w) \in \sigma^{1} \times U^{2} \\
i=1,2}}\left\|\mathrm{D}_{p} \phi^{i}(\precsim, p, w)\right\| h_{\Im}(w) \leqq \text { constant. }
\end{aligned}
$$


Here again we used $\left(\mathscr{M}_{w}\right)$. It follows that we can completely neglect the case of $(p, w)$ and $\left(p^{0}, w\right)$ both belonging to the same $H_{\S}\left(G^{i}\right)$ when we integrate local Lipschitz constants.

Let us, therefore, concentrate on the first case. Instead of integrating

$$
\sup _{p \in \tilde{U}^{1}}\left\|p-p^{0}\right\|^{-1}\left\|\int_{\tilde{U}^{2}} \phi(\lesssim, p, w)-\phi\left(\precsim, p^{0}, w\right) h_{\lessgtr}(w) \mathrm{d} w\right\|
$$

over $\lesssim$ in a neighborhood $\tilde{V}$ of $\lesssim^{0}$ we shall integrate

$$
\begin{aligned}
& \sup _{p \in \tilde{U}^{1}}\left\|p-p^{0}\right\|^{-1} \lambda^{1}\left\{w \mid(p, w) \in H_{\S}\left(G^{i}\right) \cap \tilde{U}^{1} \times \tilde{U}^{2},\right. \\
& \left.\left(p^{0}, w\right) \in H_{\Im}\left(G^{j}\right) \cap \tilde{U}^{1} \times \tilde{U}^{2}, i \neq j\right\} \\
\leqq & \sup _{p \in \tilde{U}^{1}}\left\|p-p^{0}\right\|^{-1} \lambda^{1}\left\{w \mid(p, w) \in H_{\lessgtr}\left(G^{i}\right),\left(p^{0}, w\right) \in H_{\precsim}\left(G^{j}\right), \text { for some } i \neq j\right\} \\
= & \sup _{p \in \tilde{U}^{1}}\left\|p-p^{0}\right\|^{-1} \lambda^{1}\left(\Delta^{H} \approx\left(p, p^{0}\right)\right)=\sup _{p \in \tilde{U}^{1}} Q^{H} \approx\left(p, p^{0}\right)
\end{aligned}
$$

over $\lesssim \in \tilde{V}$.

The integration makes use of $\left(\mathscr{M}_{p}\right)$. Let $d$ be some metric on the Polish space $\mathscr{P}$. Let $\eta$ be such that $B_{3 \eta}\left(\lesssim^{0}\right) \subset V$. The mapping $\mathscr{P} \times S \rightarrow \mathbb{R}$, $(\precsim, q) \mapsto d\left(\precsim, \S_{q}\right)$, is continuous. Since supp $\mu^{\prime}$ is a compact subset of $\mathscr{P}$, there is a compact symmetric neighborhood $\bar{B}=(\bar{B})^{-1}$ of id $\in S$ such that $\sup _{\precsim \in \operatorname{supp}^{\prime}} \sup _{q \in \bar{B}} d\left(\precsim, \precsim_{q}\right) \leqq \eta$. Furthermore, $\bar{B}$ is assumed to be so small that

$$
\tilde{U}^{1} \circ \bar{B}=\left\{p \circ q \mid p \in \tilde{U}^{1}, q \in \bar{B}\right\} \subset U^{1} .
$$

We shall use the disintegration associated with $\bar{B}$ according to the definition of uniform, local price dispersion.

Put $m(\precsim)=\sup _{p \in \tau_{1}} Q^{H} \precsim\left(p, p^{0}\right)$. Then

$$
\begin{aligned}
& \mathscr{J}=\int_{\tilde{V}} m(\lesssim) \mathrm{d} \mu(\lesssim)=\int_{\mathscr{P}} 1_{\tilde{V}}(\precsim) m(\precsim) \mathrm{d} \mu(\precsim) \\
& =\iint_{\mathscr{P}} \int_{\mathscr{P}} 1_{\mathfrak{V}}\left(\nwarrow^{\prime}\right) m\left(\lesssim^{\prime}\right) \mathrm{d} \xi_{\precsim}\left(\nwarrow^{\prime}\right) \mathrm{d} \mu^{\prime}(\lesssim) \\
& =\iint_{\mathscr{P}} \int_{\bar{B}}^{1} \tilde{V}\left(\lesssim_{q-1}\right) m\left(\lesssim_{q-1}\right) \mathrm{d} \xi_{\lessgtr}^{\prime}(q) \mathrm{d} \mu^{\prime}(\lesssim) .
\end{aligned}
$$

Let $\tilde{V}$ be so small that $\tilde{V} \subset B_{\eta}\left(\nwarrow^{0}\right)$. If $\lesssim \in \operatorname{supp} \mu^{\prime}, q \in \bar{B}$, then $d\left(\precsim_{q_{q}}\right)<\eta$. If, in addition, $\precsim_{q} \in \tilde{V} \subset B_{\eta}\left(\nwarrow^{0}\right)$, then 


$$
d\left(\precsim, \S^{0}\right) \leqq d\left(\precsim, \precsim_{q}\right)+d\left(\precsim_{q}, \precsim^{0}\right)=2 \eta .
$$

Hence, $\mathscr{J}<\infty$, if

$$
\begin{aligned}
& \sup _{\precsim \in \operatorname{supp} \mu^{\prime}} \int_{\bar{B}} 1_{V}\left(\precsim_{q^{-1}}\right) m\left(\lesssim_{q^{-1}}\right) \mathrm{d} \xi_{\S}^{\prime}(q) \\
& \leqq \sup _{\lessgtr \in \operatorname{supp} \mu^{\prime} \cap B_{2 \eta}\left(\Im^{0}\right)} \int_{\bar{B}} m\left(\precsim_{q^{-1}}\right) \mathrm{d} \xi_{\lessgtr}^{\prime}(q)<\infty,
\end{aligned}
$$

because $\mu$ is a finite (probability) measure. Moreover, the measures $\xi_{\lessgtr}^{\prime}$ have densities with respect to $\chi$ and hence with respect to Lebesgue measure $\lambda^{l-1}$ which are uniformly bounded. Therefore, $\mathscr{J}<\infty$, if

$$
\sup _{\preccurlyeq \in \operatorname{supp} \mu^{\prime} \cap B_{2 \eta}\left(\aleph^{0}\right)} \int_{\bar{B}} m\left(\lesssim_{q}\right) \lambda^{l-1}(\mathrm{~d} q)<\infty .
$$

Now we exploit the fact that $i\left(\Gamma_{\Im_{q}}\right)$ and $i\left(\Gamma_{\preceq}\right)$ have the same shape up to the action of $q$ on the price space. More precisely,

$$
\Delta^{H} \preccurlyeq\left(p, p^{0}\right)=\Delta^{H} \lessgtr\left(p \circ q, p^{0} \circ q\right) .
$$

Also, $\quad\left\|p-p^{0}\right\|=\left\|\left(p \circ q-p^{0} \circ q\right) \circ q^{-1}\right\|$. Therefore, for $q^{\prime} \in \bar{B}, \quad \precsim \in \operatorname{supp} \mu^{\prime}$ $\cap B_{2_{\eta}}\left(\nwarrow^{0}\right)$,

$$
\begin{aligned}
Q^{H} \precsim q\left(p, p^{0}\right) & \leqq \sup _{p \in \tilde{U}^{1}} \sup _{q \in \bar{B}}\|q\| Q^{H} \preccurlyeq\left(p \circ q, p^{0} \circ q\right) \\
& \leqq \text { const } \cdot \sup _{p \in \tilde{U}^{1}} Q^{H} \preccurlyeq\left(p \circ q, p^{0} \circ q\right) .
\end{aligned}
$$

Hence it suffices to show that $\int_{\vec{B}} \sup _{p \in \tilde{U}^{1}} Q^{H} \preccurlyeq\left(p \circ q, p^{0} \circ q\right) \lambda^{l-1}(\mathrm{~d} q)$ has a bound independent of $\lesssim \in \operatorname{supp} \mu^{\prime} \cap B_{2 \eta}\left(\lesssim^{0}\right)$.

Finally, this bound is obtained with the aid of Lemmas 4 and 6. Since $\operatorname{supp} \mu^{\prime} \cap B_{2 \eta}\left(\Xi^{0}\right)$ is compact, there exists a compact cube $C$ in $\mathbb{R}^{l-1}$ parallel to the axes and centered at the origin such that $U^{1} \subset H_{\lessgtr}^{1}(C)$ for all $\precsim \in \operatorname{supp} \mu^{\prime} \cap B_{2 \eta}\left(\nwarrow^{0}\right)$. By Lemma 4 and the change of variable formula for integrals it suffices to show that

$$
\int_{C} \sup _{\hat{x} \in C} Q\left(\hat{x}, \hat{x}^{0}\right) \lambda^{l-1}\left(\mathrm{~d} \hat{x}^{0}\right)<\infty,
$$

since the determinant of $\mathrm{D}\left(H_{\breve{0}}^{1}\right)(\hat{x})$ is bounded away from zero uniformly in $\hat{x} \in C$ and $\precsim \in \operatorname{supp} \mu^{\prime} \cap B_{2 \eta}\left(\precsim^{0}\right)$. Lemma 6 implies that this integral is indeed finite.

$$
\text { Q.E.D. }
$$




\section{References}

Araujo, A. and A. Mas-Colell, 1978, Notes on the smoothing of aggregate demand, Journal of Mathematical Economics 5, 113-127.

Bauer, H., 1968, Wahrscheinlichkeitstheorie und Grundzüge der Maßtheorie (De Gruyter, Berlin).

Bröcker, T. and L. Lander, 1975, Differentiable germs and catastrophes (Cambridge University Press, Cambridge).

Debreu, G., 1972, Smooth preferences, Econometrica 40, 603-615.

Dierker, E., H. Dierker and W. Trockel, 1980a, Continuous mean demand functions derived from non-convex preferences, Journal of Mathematical Economics 7, 27-33.

Dierker, E., H. Dierker and W. Trockel, 1980b, Smoothing demand by aggregation with respect to wealth, Journal of Mathematical Economics 7, 227-247.

Golubitsky, M. and V. Guillemin, 1973, Stable mappings and their singularities (Springer, New York).

Hildenbrand, W., 1974, Core and equilibria of a large economy (Princeton University Press, Princeton, NJ).

Hildenbrand, W., 1980, On the uniqueness of mean demand for dispersed families of preferences, Econometrica 48, 1703-1710.

Mas-Colell, A., 1976, The theory of economic equilibrium from the differentiable point of view, Course notes, Mathematics Department (University of California, Berkeley, CA).

Mas-Colell, A., 1977, Regular, nonconvex economies, Econometrica 45, 1387-1407.

Mas-Colell, A., 1978, Preferences, utility and demand, manuscript (University of California, Berkeley, CA).

Mas-Colell, A. and W. Neuefeind, 1977, Some generic properties of aggregate excess demand and an application, Econometrica 45, 591-599.

Parthasarathy, K.R., 1967, Probability measures on metric spaces (Academic Press, London).

Parthasarathy, K.R., 1967, Probability measures on metric spaces (Academic Press, London).

Schwartz, L., 1967, Cours d'analyse (Hermann, Paris).

Sondermann, D., 1975, Smoothing demand by aggregation, Journal of Mathematical Economics 2, 201-223.

Sondermann, D., 1976, On a measure theoretical problem in mathematical economics, in: A. Bellow, ed., Measure theory, Lecture notes in mathematics, no. 541 (Springer, New York).

Sondermann, D., 1980, Uniqueness of mean maximizers and continuity of aggregate demand, Journal of Mathematical Economics 7, 135-144.

Yamazaki, A., 1979, Continuously dispersed preferences, regular preference-endowment distribution and mean demand function, in: J.R. Green and J.A. Scheinkman, eds., General equilibrium, growth and trade, Essays in honor of Lionel McKenzie (Academic Press, New York) 13-24. 